\title{
Animal Models of Calcific Aortic Valve Disease
}

\author{
Krista L. Sider, ${ }^{1}$ Mark C. Blaser, ${ }^{1}$ and Craig A. Simmons ${ }^{1,2,3}$ \\ ${ }^{1}$ Institute of Biomaterials and Biomedical Engineering, University of Toronto, 164 College Street, Toronto, ON, Canada M5S $3 G 9$ \\ ${ }^{2}$ Department of Mechanical and Industrial Engineering, University of Toronto, 5 King's College Road, Toronto, ON, Canada M5S $3 G 8$ \\ ${ }^{3}$ Faculty of Dentistry, University of Toronto, 124 Edward Street, Toronto, ON, Canada M5G 1 G6
}

Correspondence should be addressed to Craig A. Simmons, c.simmons@utoronto.ca

Received 29 March 2011; Accepted 27 April 2011

Academic Editor: Elena Aikawa

Copyright () 2011 Krista L. Sider et al. This is an open access article distributed under the Creative Commons Attribution License, which permits unrestricted use, distribution, and reproduction in any medium, provided the original work is properly cited.

Calcific aortic valve disease (CAVD), once thought to be a degenerative disease, is now recognized to be an active pathobiological process, with chronic inflammation emerging as a predominant, and possibly driving, factor. However, many details of the pathobiological mechanisms of CAVD remain to be described, and new approaches to treat CAVD need to be identified. Animal models are emerging as vital tools to this end, facilitated by the advent of new models and improved understanding of the utility of existing models. In this paper, we summarize and critically appraise current small and large animal models of CAVD, discuss the utility of animal models for priority CAVD research areas, and provide recommendations for future animal model studies of CAVD.

\section{Introduction}

Valvular heart diseases account for over 23,000 deaths annually in the United States, with the aortic valve being the most frequently affected [1]. The aortic valve is composed of three semilunar cusps or leaflets that passively open and close over 25 million times per year to maintain unidirectional blood flow from the left ventricle to the systemic and coronary circulations. To meet its functional requirements under these demanding conditions, the thin, pliable leaflet tissue is organized into three distinct layers: (1) the fibrosa on the aortic side of the leaflet, composed primarily of collagen; (2) the spongiosa in the middle, composed mainly of proteoglycans; and (3) the ventricularis on the ventricular side, composed of collagen and elastin [2]. The cellular components of the aortic valve include a monolayer of valvular endothelial cells (VECs) on the outer surface of the leaflets and valvular interstitial cells (VICs), which populate each of the three layers of the leaflet. VICs are a heterogeneous population of mostly fibroblasts [3], a subpopulation of which are mesenchymal progenitor cells [4]. VICs play the critical role of remodelling and organizing the valve extracellular matrix $(\mathrm{ECM})$ to maintain valve integrity $[3,5]$.

In disease, ECM structure and organization are disturbed, resulting in valve dysfunction. The most common valvular disease is calcific aortic valve disease (CAVD) [1]. CAVD encompasses early sclerosis, characterized by leaflet thickening without left ventricular outflow obstruction, to late stenosis in which leaflets stiffen, flow is obstructed, and cardiac function is compromised. The consequences of CAVD are significant: sclerosis is associated with a $50 \%$ increased risk of cardiovascular death and myocardial infarction [6], and the prognosis for patients with stenosis is very poor [7]. Because CAVD is a slowly progressing disease taking several decades to develop, it was once thought to be a passive "degenerative" or "senile" process. However, CAVD is now recognized to be an active pathobiological process that shares many risk factors with atherosclerosis, including hypercholesterolemia, smoking, hypertension, diabetes, chronic renal disease, and male gender [8-10].

Also similar to atherosclerosis, chronic inflammation is a predominant feature of CAVD. This is reflected in human disease by the presence of macrophages and $\mathrm{T}$ cells [1116]; subendothelial oxidized low-density lipoprotein (LDL) deposits [11-13, 16, 17]; elevated superoxide and hydrogen peroxide $[18,19]$; active mast cells $[17,20]$; complement activation [16]; elevated expression of tumor necrosis factor- $\alpha$ $($ TNF- $\alpha$ ) [14], matrix metalloproteinases (MMP-1, -2, -3, -9) [14, 21], interleukin-2 (IL-2) [22], angiotensin converting enzyme (ACE), angiotensin II (AngII), angiotensin II type-1 
receptor (AT1R), and chymase [20, 23]; and VEC expression of intracellular adhesion molecule-1 (ICAM-1), vascular cell adhesion molecule-1 (VCAM-1) [24, 25], and E-selectin [25]. Inflammatory processes are associated with, and may drive, the alterations in the valve ECM that are hallmarks of CAVD, including leaflet thickening [13, 14, 21]; collagen turnover and fibrosis $[13,24,26]$; accumulation of proteoglycans and hyaluronan [27, 28]; fragmentation of elastin $[29,30]$; and calcification [11-13, 15, 17, 22, 24]. Ultimately, it is maladaptation of the valve ECM (which is often localized to the fibrosa layer $[11,13])$ that results in valve stiffening and dysfunction.

While many features of human CAVD are well described (particularly for late-stage disease), specific pathobiological mechanisms are not fully understood. Some insight may come from similarities with atherosclerosis, but less than $40 \%$ of patients with CAVD have clinically significant coronary atherosclerosis [31], suggesting distinct processes. Thus there is an unmet scientific need to determine pathobiological mechanisms of CAVD and identify new approaches to treat CAVD. Animal models are emerging as vital tools to this end, facilitated by the advent of new models and improved understanding of the utility of existing models. In this paper, we summarize and critically appraise current small and large animal models of CAVD, discuss the utility of animal models for priority CAVD research areas, and provide recommendations for future animal model studies of CAVD.

\section{Animal Models of CAVD}

Animal models are an important platform for studying the initiation and progression of CAVD in vivo, as well as for judging the effectiveness of therapeutic interventions. To be most effective, models should mimic human disease or at least important facets thereof and the conditions in which human CAVD develops. The most common species used to model CAVD are mouse, rabbit, and swine. Of these, only swine develops CAVD naturally with age, but this process is slow and is usually accelerated by diet-induced hypercholesterolemia. Rabbits are not naturally susceptible to CAVD but are responsive to diet-induced hypercholesterolemia, and mice require a genetic predisposition to promote advanced disease.

2.1. Mouse Models. In the study of CAVD, a large proportion of contemporary animal work is performed in mouse models, as mice are uniquely suited to mechanistic studies of the root causes of aortic valve pathology. Mouse models of CAVD offer a number of advantages, including their small size, easy husbandry, and cost effectiveness. In addition, short generation time, resultant ease of genetic manipulations, and availability of clonal samples allow specific investigation of key molecular mediators of CAVD $[32,33]$. A summary of the critical elements of mouse models of CAVD is presented in Table 1.

Despite these advantages, mouse models do suffer from several important limitations. Firstly, the anatomical structure of mouse aortic valves differs drastically from that of humans. Mice do not have the trilayer aortic valve tissue morphology characteristic of human, pig, or rabbit valves; rather their leaflets are usually only $\sim 5-10$ cells thick and do not exhibit segregated layers [62]. Secondly, and most importantly, wild-type (WT) mice on standard diets do not exhibit spontaneous calcification [32], and consequently the study of CAVD in mouse models requires dietary [34] and/or genetic $[34,38,42,43]$ or other interventions [46, 47] to induce a valvular calcium burden.

Furthermore, the development of atherosclerosis in inbred mice is dependent on the background strain in use. When comparable hyperlipidemia is induced in C57BL/6J mice, this strain exhibits considerably greater atherosclerotic lesion formation than the FVBN or C3H strains [63-65]. While strain-specific susceptibility to CAVD has not been explored in detail, similarities between atherosclerosis and valve disease imply that comparable strain-specific susceptibility to CAVD is present as well. In a related issue, while the $\mathrm{C} 57 \mathrm{BL} / 6 \mathrm{~J}$ mouse is the most atheroprone strain and most commonly used background in mouse models of CAVD, their valve leaflets contain black pigmentation that may be melanocytes [66] or lipofuscin-containing granules [35]. Regardless of their identity, these dark particulates can appear in the valve interstitium and are easily mistaken as positive von-Kossa calcification staining, thus requiring the use of alizarin red to accurately determine the extent of calcification in C57BL/6J models of CAVD [62].

2.1.1. Diet-Induced and Genetic Mouse Models. As WT mouse strains do not spontaneously calcify, dietary and/or genetic insults are often employed. Most commonly, mice deficient in the low-density lipoprotein (LDL) receptor $(L d l r-/-)$ are used. Ldlr-/- mice with only apolipoprotein B (ApoB)-100 ( $L d l r-/-; A p o b^{100}$-only) spontaneously develop mild hypercholesterolemia $(\sim 270 \mathrm{mg} / \mathrm{dL})$, drastic reductions in valve orifice diameter $(>50 \%)$, elevated transvalvular systolic pressure gradients, and left ventricular hypertrophy when aged for 17-22 months without dietary insult [38]. Furthermore, ageing is associated with superoxide development indicative of the onset of oxidative stress and abundant von Kossa staining in the aortic valve [38].

Typically, the Harlan Teklad TD.88137, or "Western" diet, is used in models with a dietary induction component. This adjusted diet derives $21.2 \%$ weight and $42 \%$ of total calories from fat, with $0.2 \%$ [36, 42-44, 46] or $0.25 \%[39,40]$ cholesterol (mouse diet fat content is typically expressed as percent of total calories from fat). Towler and colleagues first reported extreme hyperlipidemia $(\sim 1040 \mathrm{mg} / \mathrm{dL})$, hyperinsulinemia/hyperglycemia, and mineral deposition in $L d l r-/-$ mice fed TD.88137 for 16 weeks [36]. Ldlr-/- mice fed a similar high-cholesterol diet $(0.15 \%)$ develop increases in valve thickness, macrophage accumulation, superoxide production, activated myofibroblasts and osteoblasts, and mineralization [37]. When mice are regularly exercised while the high-cholesterol diet is fed, these pathological symptoms are significantly reversed [37]. The Ldlr-/-;Apob ${ }^{100}$-only mouse has also been modified to incorporate a conditional knockout of the microsomal triglyceride transfer protein $(M t t p)$ under the control of an 


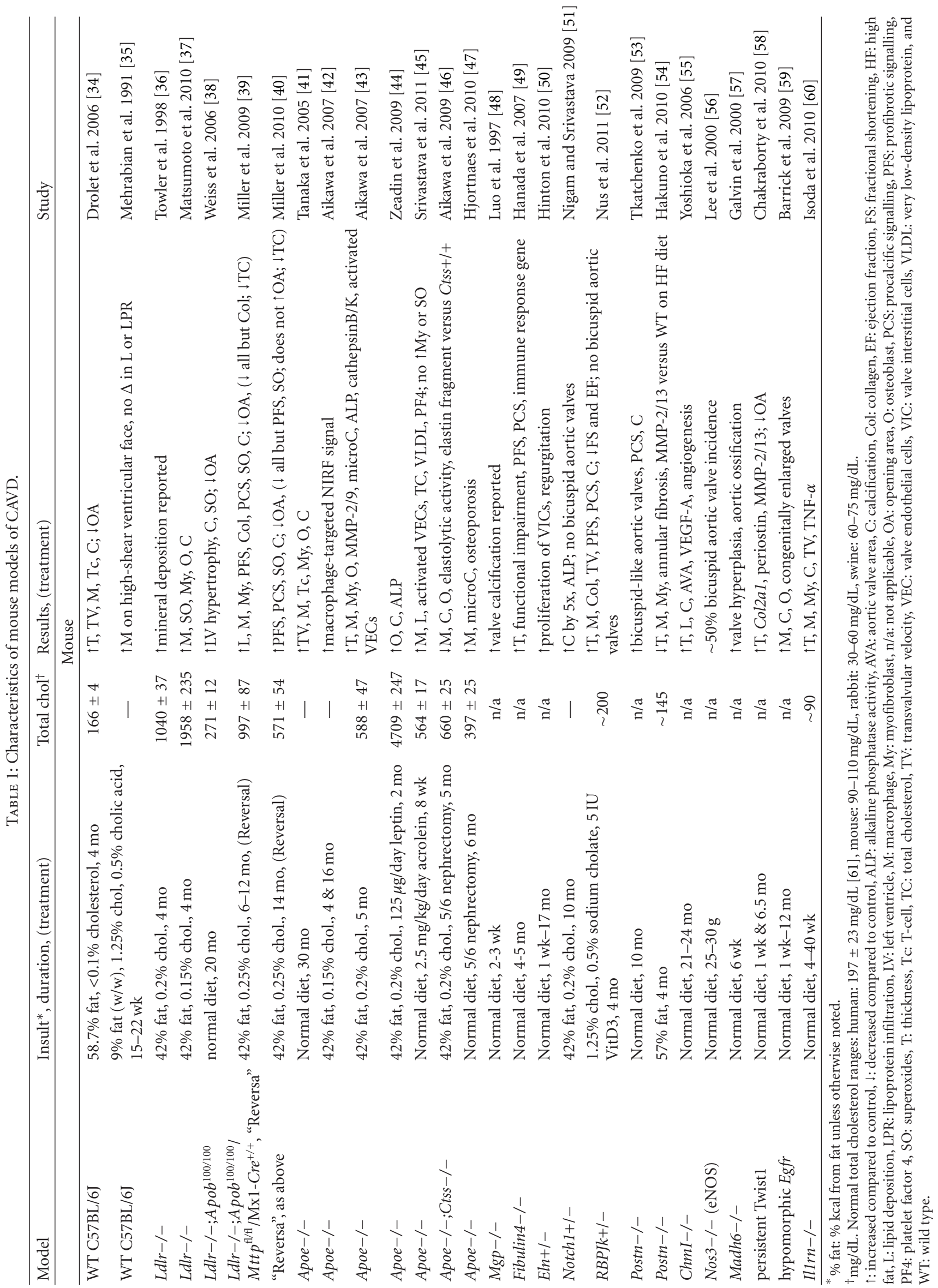


inducible Mx1-Cre transgene. Known as the Reversa model,

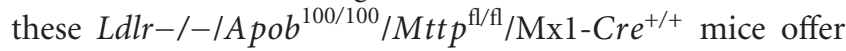
Cre-mediated loss of Mttp activity (required for ApoB lipoprotein secretion) and allow controllable normalization of serum cholesterol levels [39]. In conjunction with the TD.88137 diet, the Reversa mouse develops robust calcific aortic stenosis. Serum cholesterol rises to $800-1000 \mathrm{mg} / \mathrm{dL}$ in 6-12 months, and lipid deposition and macrophage infiltration are significantly increased. Profibrotic signalling and myofibroblast activation as measured by pSmad 2 and $\alpha$-smooth muscle actin ( $\alpha \mathrm{SMA})$ are elevated, as is procalcific signalling (pSmad1/5/8, Msx2, $\beta$-catenin, Runx2, osterix) and superoxide levels, leading to oxidative stress, valvular mineralization, and positive von Kossa/alizarin red staining [39, 40]. Importantly, Cre-mediated loss of Mttp activity at six months ("reversal") normalizes serum cholesterol levels, decreases valvular lipid deposition and macrophage infiltration, prevents further calcification, lowers $\mathrm{pSmad} 2 / \alpha \mathrm{SMA}$, lowers $\mathrm{pSmad} 1 / 5 / 8 / \mathrm{Msx} 2 / \mathrm{Run} \times 2$, attenuates oxidative stress, and results in functional improvements in cusp separation [39]. Interestingly, reversal after 12 months does not lower pSmad2 or superoxide levels and does not improve leaflet cusp separation distance [40]. The on-demand "switching" of cholesterol levels in this model allows, for the first time, regression studies in dietary-induced CAVD independent of pharmacological intervention.

Diets with substantially elevated cholesterol levels are, however, not always employed as initiators of mouse CAVD. One such study employed the use of a high-fat, high-carbohydrate diet, where $35.5 \%$ of weight and $59 \%$ of calories were derived from fat, but no cholesterol was added $(<0.1 \%$ present $)$ [34]. In WT C57BL/6J mice on this diet, total cholesterol levels are mildly elevated to $166 \mathrm{mg} / \mathrm{dL}$, while $L d l r-/-$ mice exhibit drastic hypercholesterolemia at $722 \mathrm{mg} / \mathrm{dL}$ and develop overt diabetes mellitus. Most interestingly, the high-fat, low-cholesterol diet is able to induce early markers of CAVD even in WT mice: thickened leaflets, black particulates which may be von-Kossa-positive calcification, decreased aortic valve opening area, and increased transvalvular blood velocities are reported, along with CD68positive macrophage and T-lymphocyte infiltration into the valvular interstitium. These macrophages infiltrate primarily on the high-shear ventricular side of the mouse valve [35]. The development of these disease hallmarks in WT mice with only mild hypercholesterolemia may prove to be more relevant to human CAVD.

In addition to the Ldlr-/- model, a second common genetically manipulated model is the endogenously hyperlipidemic [63] ApoE-deficient (Apoe-/-) mouse [41-44, 46, 47]. ApoE allows receptor-mediated removal of very-low density lipoprotein (VLDL) from the circulation. However, ApoE also regulates T-cell proliferation and macrophage function and modulates lipid antigen presentation as well as general levels of inflammation and oxidation [67]. In this way, deletion of Apoe may significantly impact the inflammatory response to CAVD in a manner distinctly unrelated to hypercholesterolemia and/or pathogenesis of the human disease. This potential for differential disease progression remains to be studied in detail. Without dietary intervention, Apoe-/- mice develop hypercholesterolemia $(\sim 490 \mathrm{mg} / \mathrm{dL})$ [68], and their increasing age up to 2.5 years is correlated with increases in transvalvular velocity, mild aortic regurgitation, $\alpha$ SMA and osteocalcin $(\mathrm{OCN})$ expression, macrophage and T-cell infiltration, and nodular calcification [41]. Administration of the TD.88137 diet to Apoe-/- mice for 4-5 months induces accelerated early disease with a substantial increase in serum cholesterol to $\sim 588 \mathrm{mg} / \mathrm{dL}$, thickened leaflets, activated endothelial cells, and subendothelial lesions rich in macrophages (colocalized with MMP-2/9, cathepsin B, $\alpha$ SMA, ALP, Runx2, and OCN expression) $[42,43]$. Importantly, there is no evidence of von Kossa or alizarin red staining at this early time point, though a bisphosphonate-conjugated imaging particle shows signs of early microcalcification and colocalizes with cathepsin $\mathrm{K}$ $[42,43]$. Another version of the Apoe-/- model includes coadministration of the adipocytokine leptin, a known cardiovascular risk factor [44]. Leptin treatment does not induce hypercholesterolemia nor does atherosclerotic lesion size change, but von Kossa and ALP-positive staining is significantly increased in leptin-treated Apoe-/- mice, and osteopontin (OPN) and OCN expression is also increased. While on a normal diet, oral exposure of Apoe-/- mice to acrolein, a dietary aldehyde generated during inflammation and oxidative stress, induces hypercholesterolemia, macrophage and lipid infiltration, and platelet and endothelial activation [45]. Interestingly, such treatment does not induce fibrosis, nor does it provoke a systemic oxidative stress response.

Along with these two common hyperlipidemic models, there exist other interesting genetic models that recapitulate some aspects of CAVD. Knockout of the mineral binding ECM protein matrix GLA protein $(M g p-/-)$ produces spontaneous ectopic apatite formation in the arterial collagen fibrils and von-Kossa-positive calcification in the aortic valve [48]. An insufficiency of elastin (Eln+/-) produces proliferation of VICs and aortic regurgitation [50]. Hypomorphic expression of fibulin-4, an ECM stabilizing protein, results in thickened leaflets, significant functional impairments, and positive $\mathrm{pSmad} 2$, pSmad1/5/8, and von Kossa staining [49]. Mutant tissue displays increased transforming growth factor- $\beta$ (TGF- $\beta$ ) and bone morphogenic protein (BMP) signalling, while an Affymetrix microarray showed differential expression of a number of immune response genes between the WT and fibulin- 4 deficient animals. Of specific interest to the role of inflammation in spurring the onset of CAVD are mice deficient in the antiinflammatory cytokine interleukin-1 receptor antagonist (Il1 $n-/-$ ). These animals develop thickened aortic valves infiltrated by macrophages and containing differentiated myofibroblasts, while aged Ill $\mathrm{rn}-/$ - mice develop calcified lesions with functional impacts on transvalvular blood velocity [60]. Circulating levels of TNF- $\alpha$ rise dramatically in this model, and the importance of this chemokine to CAVD pathogenesis is underscored by double knockout TNF- $\alpha-/-; I l 1 r n-/-$ mice which do not develop CAVD [60]. 
2.1.2. Congenital and Developmental Mouse Models. The presence of a congenital bicuspid aortic valve is associated with drastically increased risk of CAVD [69]. Mutations in the transcriptional regulator NOTCH1 have been shown to cause bicuspid valves and CAVD development in humans. The normally developing mouse valve displays higher Notch1 levels than during postnatal growth [70]. Mice heterozygous for Notch1 (Notch1+/-) fed a Western diet with $0.2 \%$ cholesterol for 10 months exhibit fivefold greater aortic valve calcification than WT controls, but do not exhibit bicuspid valves [51]. Recently, mice haploinsufficient for the primary nuclear Notch effector protein recombining binding protein suppressor of hairless (RBPJK) were challenged with a high-cholesterol/cholate diet for a shorter 4-month period [52]. These mice present normal trileaflet aortic valves, and develop thickened and calcified leaflets, macrophage infiltration, collagen deposition, and profibrotic/osteogenic signalling. Interestingly, Notch1+/- mice display relatively little functional impairment when compared to RBPJk+/mice, implying that other Notch effectors contribute to valvular homeostasis [52]. Periostin is highly expressed in the endocardial cushion during embryogenesis, and its deletion (Postn-/-) induces overexpression of delta-like 1 homolog (Dlk1), a negative regulator of Notch1 [53]. By 10 months of age, the valves of Postn-/- mice exhibit a severely deformed bicuspid-like morphology displaying expression of Runx2, OPN, and OCN, along with significant valvular calcification (von Kossa). Paradoxically, when Postn-/mice are fed a high-fat diet for four months, they display decreased valve thickness, macrophage infiltration, myofibroblast differentiation, annular fibrosis, and MMP-2/13 expression when compared with WT mice fed the same diet, possibly reflecting a reduced ability of myofibroblasts and macrophages to adhere to and infiltrate the ECM [54]. Periostin expression is mutually exclusive to that of chondromodulin-I (ChmI), an antiangiogenic factor. Aged ChmI-/- mice display increases in valve thickness, lipid deposition, calcification, VEGF-A, and angiogenesis [55]. In humans, expression of endothelial nitric oxide synthase (eNOS) in the valvular endothelium is drastically reduced in bicuspid valves [71], and mice lacking eNOS (Nos3-/-) display a high incidence $(\sim 50 \%)$ of bicuspid aortic valve [56]. The susceptibility of this mouse model to CAVD and inflammatory processes is as of yet unexplored. Paradoxically however, Nos3-/- mice do not develop atherosclerosis when fed a high-cholesterol atherogenic diet [72], a phenomenon which may be the result of reductions in eNOS-driven LDL oxidation in the vasculature [73].

Signalling pathways normally associated with embryonic development of the valve have recently been implicated in CAVD pathogenesis, and have become the focus of several mouse models of the disease [5]. While epidermal growth factor receptor (EGFR) has been implicated in the development of cancer, and targeted for inhibition by cancer therapeutics, mice carrying a hypomorphic allele of $E g f r$ $\left(E g f r^{\text {wa2/wa2 }}\right.$ ) exhibit congenitally enlarged valves, and mature mice display valvular OPN expression, macrophage infiltration, and extensive von Kossa-positive calcification [59]. Interestingly, these results imply that cancer patients with congenital valve defects or other cardiovascular risk factors should avoid EGFR inhibition. The Smad6 inhibitory protein regulates TGF- $\beta$ signalling and mediates endocardial cushion transformation, and mutation of the Smad6 gene (Madh6-/-) in mice produces valvular hyperplasia, outflow tract septation defects, elevated blood pressure, and aortic ossification [57]. Endocardial cushion development is also promoted by the Twist1 transcription factor and is upregulated in human CAVD. Mice engineered with persistent Twist1 (CAG-CAT-Twist1;Tie2Cre mice) develop two- to threefold increases in area, length, and thickness of the aortic valve [58]. Expression of collagen-II, periostin, and the matrix remodelling enzymes MMP-2/13 are also elevated [58].

2.2. Rabbit Models. In CAVD research, rabbits are the most common large animal model used. Rabbits are desirable for a number of reasons as they (1) have a trilayer valvular morphology [74]; (2) respond to dietary cholesterol [74-86]; (3) are relative easy to manage due to their medium size; (4) have some similarities to human lipoprotein metabolism [87-89]; (5) are susceptible to accelerated calcification with vitamin D2 (VitD2) [80, 82]; (6) are available as transgenic [90-92] and natural mutant strains $[93,94]$.

On the other hand, there are a number of dissimilarities with human disease. Rabbits do not form spontaneous atherosclerotic lesions and therefore require very high cholesterol levels to induce more advanced disease [78-80, 84, 85], unless very long-term studies $[74,83,86]$ or VitD2 supplementation [80, 82] are used. Rabbits also have significant differences in their lipid metabolism from humans [87-89], which can result in their development of cholesterol storage syndrome while on high-cholesterol diets (0.5-3\%), with cholesterol deposited in regions such as the liver, adrenal cortex, and reticuloendothelial and genitourinary systems. Rabbits have also been reported to form atherosclerotic lesions that do not resemble those in humans [74, 88, 102]. A summary of the critical elements of rabbit models of CAVD is presented in Table 2 .

2.2.1. Diet-Induced Rabbit Models. The standard rabbit model is the New Zealand white rabbit (NZWR) with a starting weight of 1.6-3 kg [74, 76-86] and hypercholesterolemia induced by one of four main diet categories (1) moderate-tohigh cholesterol with $[75-77,81]$ or without fat $[78,79,84$, 85, 95, 103]; (2) moderate cholesterol and VitD2 [19, 80, 82]; (3) low cholesterol [74, 83, 86]; or (4) VitD2 only [82, 98]. (Below, rabbit diet additions are expressed as additional weight percentage added.)

Moderate-to-high cholesterol diets (0.5-2\%) induce very high total blood cholesterol levels, ranging from approximately 1000-3500 mg/dL [79, 80, 84, 85], and sometimes induce extreme hypertriglyceridemia [79]. After one week on this diet, lipids infiltrate into the subendothelial region of the fibrosa prior to macrophage presence [75] and associate with collagen fibres and the proteoglycans that connect them $[76,81]$. This is paralleled by hyperplasia of the basal lamina, including fragmentation of the elastin and collagen bundles, and accumulation of proteoglycan in the fibrosa [75], with 


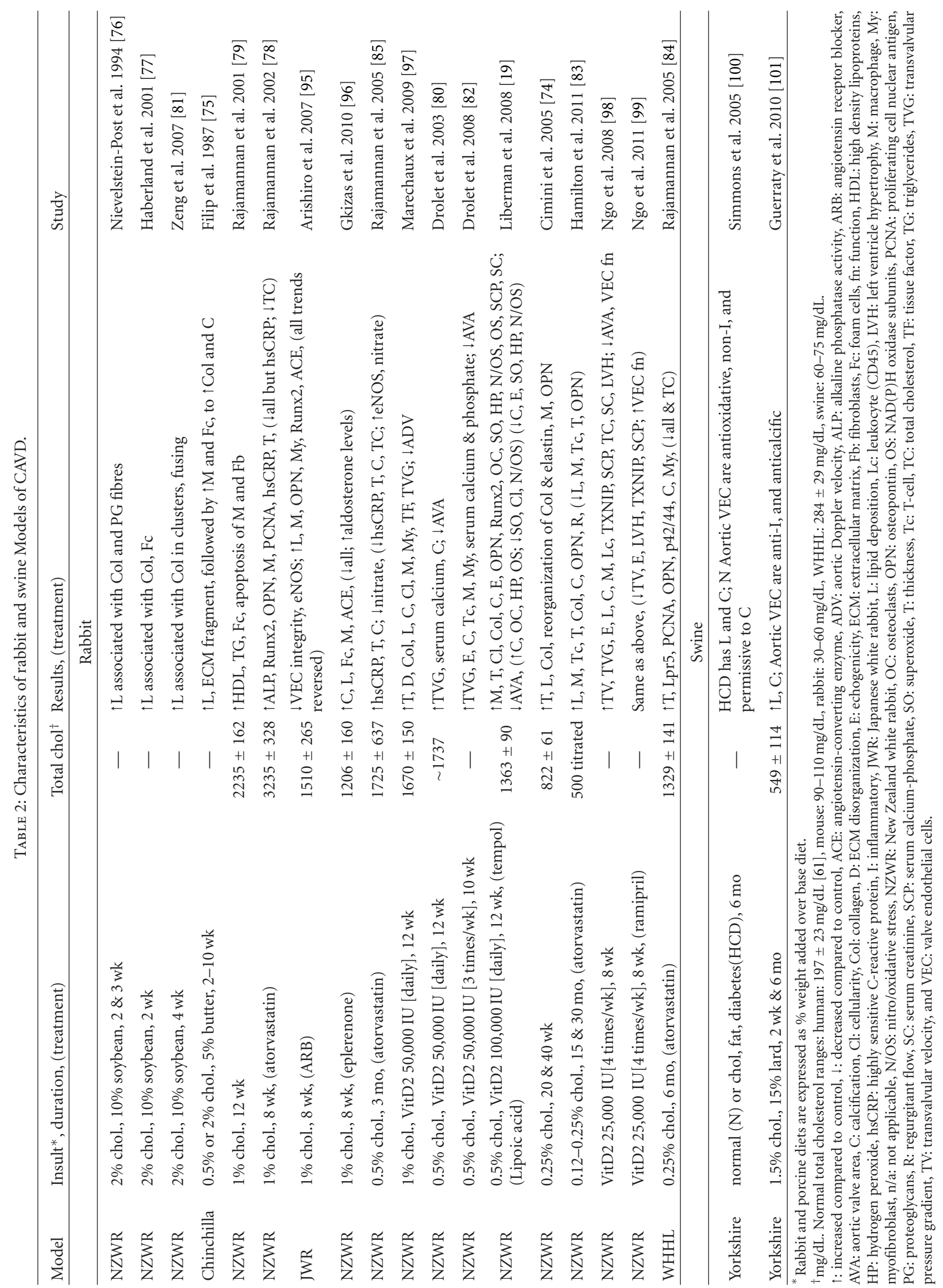


both VECs and VICs increasing their protein production. By eight weeks there is macrophage infiltration, myofibroblast presence, and increased proliferation, ACE, OPN, and osteoblast gene expression (alkaline phosphatase, OPN, and Runx2) [78, 95]. Highly sensitive C reactive protein (hsCRP) in the blood is also increased, indicating an inflammatory state [78], and there is impaired endothelial integrity on the aortic side with decreased eNOS expression [95]. Foam cells and lipid uptake by VICs have also been seen prior to 12 weeks [75]. By 12 weeks, some calcification is present [85], and there is a slight increase in apoptosis of macrophages and proliferating fibroblasts $(0.1 \%$ of cells) [79]. With the addition of VitD2 to $0.5-1 \%$ cholesterol diets, by $10-12$ weeks there is enough calcification and thickening to cause decreased aortic valve opening area [19, 80, 82] and aortic Doppler velocity index [97], and increased transvalvular velocities [80, 82, 97] and echogenicity [19]. There are also macrophages present in the subendothelial spaces $[19,82$, 97], and $T$ cells [82] and smooth muscle actin positive cells found near the calcification $[82,97]$. These valves are thickened due to increased collagen, cellular infiltrates, calcification [19, 97], and lipid deposition [97] in the fibrosa, with increased expression of OPN and Runx2, and the presence of osteoclasts. Reactive oxygen species (ROS) are also present around calcified areas (superoxides, hydrogen peroxide, indicators of nitro/oxidative stress and $\mathrm{NAD}(\mathrm{P}) \mathrm{H}$ oxidative subunits) [19], as well as tissue factor [97].

Low-cholesterol diets $(0.125-0.25 \%$ cholesterol) maintain more moderate cholesterol levels of around 500$800 \mathrm{mg} / \mathrm{dL}[74,83,86]$. By five months, lipid deposition and collagen disarray are seen in the fibrosa, with macrophages and OPN in the lesion. By 10-15 months there is an increase in macrophages, OPN, collagen area, thickness, and lipid deposition. However, no calcification is seen $[74,83]$ until 30 months, which is accompanied by an increase in macrophages, $\mathrm{T}$ cells, and collagen deposition, and the presence of regurgitant flow [83].

VitD2-only treatment has been used in some models to induce advanced CAVD $[82,98]$. By 8-10 weeks, functional changes indicative of mild stenosis including increased peak transvalvular velocity and pressure gradients, increased echogenicity of the valve $[82,98]$, and decreased aortic valve area [98] occur. Histologically, these valves display increased burden of lipids, leukocytes, macrophages [98], myofibroblasts, $\mathrm{T}$ cells [82], and calcification [82, 98]. Serum levels of cholesterol, calcium, phosphate, creatinine [98], and calcium-phosphate product [82] were also seen to increase. There is also the indication that VitD2 might increase the oxidative stress (increased thioredoxin-interacting protein) and impair VEC function (increased plasma asymmetric dimethylarginine, an NO inhibitor) [98]. This indicates that VitD2 alone is capable of inducing CAVD resulting in functional impairment of the valve [98]. However, use of a hypercholesterolemic diet in combination with VitD2 at 12 weeks produces greater valvular functional impairment than VitD2 alone [82].

2.2.2. Genetic Rabbit Models. Rabbit models utilizing spontaneous mutations as well as transgenic manipulations are available. These models primarily have alterations in the LDLR and/or apolipoproteins that result in hypercholesterolemia when on a cholesterol-free, limited fat diet. Such models include (1) Watanabe heritable hyperlipidemic (WHHL) rabbits, which have a spontaneous LDLR mutation [84, 93, 104, 105]; (2) St. Thomas Hospital rabbits, which acquire hypertriglyceridemia as well as hypercholesterolemia [94]; (3) rabbits with altered lipid profiles, such as induced human ApoB100 [90, 94] or Apo(a) [91]. Of these, only the WHHL rabbit has been used in a study of CAVD to show that hypercholesterolemia-induced calcification may be mediated in part by the LPR5/ $\beta$-catenin pathway [84]. Comprehensive reviews of genetically altered models of rabbit atherosclerosis have been done by Brousseau and Hoeg [87] and Fan and Watanabe [92].

2.3. Porcine Models. Porcine models are regarded as excellent animals for atherosclerosis research $[88,89,106-108]$ and more recently for the study of CAVD [32]. Swine have many similarities to humans, including similar systemic hemodynamic variables and heart anatomy [89], including trilayered aortic valve leaflets. They also have similar lipid profiles [61] and lipoprotein metabolism [106, 108] to humans, though their high-density lipoprotein (HDL) level does rise with hypercholesterolemic diets. The porcine genome is of a comparable size to that of humans and is homologous in both sequence and chromosomal structure [32]. Swine naturally develop atherosclerotic lesions, which are accelerated by high-cholesterol/high-fat diets and result in human-type lesions [108-111]. The size of swine also makes them ideal for studies that characterize leaflet mechanical properties and for studies requiring blood analysis.

Size is also the primary limitation of swine, as there is increased complexity and expense in maintaining them. In many cases, this has led to the use of mini- $[106,112,113]$ and micro- [114] swine breeds for atherosclerosis studies, instead of full sized Yorkshire swine. Standard weights at six months are around $33 \mathrm{~kg}$ for Yucatan mini, $24 \mathrm{~kg}$ for Sinclair mini, and $20 \mathrm{~kg}$ for Yucatan microswine. These are significantly smaller than Yorkshire swine (approximately $115 \mathrm{~kg}$ at six months). Smaller breeds also develop human-type atherosclerosis lesions ranging from early (3-4 months) [106, 113] to advanced lesion ( 8 months) with a necrotic core, fibrous cap, haemorrhage, calcification, and medial thinning [112]. They may also be very good models for CAVD investigations, but have not been used for this purpose to date. A summary of the critical elements of porcine models of CAVD is presented in Table 2.

2.3.1. Diet-Induced Porcine Models. Though porcine models have been principally used in atherosclerosis research, they have recently been employed to study CAVD. Typical hypercholesterolemic swine diets consist of a standard corn/soybean diet with an additional 1.5-2\% cholesterol and 10-20\% fat, sometimes with $0.7-1.5 \%$ sodium cholate (porcine diet additions are expressed as additional weight percentage added). There is some indication that diets started prior to sexual maturity may be more effective at producing advanced disease [108]. Standard lipid profiles for studies ranging 
from 2 weeks to 12 months in length show total cholesterol of $\sim 300-500 \mathrm{mg} / \mathrm{dL}$ (2- to 8-fold increase), LDL $\sim 200$ $500 \mathrm{mg} / \mathrm{dL}$ (4- to 11-fold increase), HDL increases of 1.5to 4 -fold, and heterogeneous triglyceride (TG) levels ranging from twofold increase to twofold decrease.

Initial CAVD studies show evidence that swine on hypercholesterolemic diets develop human-type disease. Macroscopic focal areas of increased opacity are seen by six months on a hypercholesterolemic diet with [100] or without diabetes [101]. Small early calcific nodules are also seen histologically at 6-7 months of age either with diet for two weeks or six-months [101] and with six month diet and diabetes [100]. Subendothelial lipid infiltration is seen only within the fibrosa layer, increasing with diet duration [100, 101]; however there is no frank inflammation seen after two weeks or six months of diet [101]. In normal valves it has been found that the VECs on the aortic side of the valve have an antioxidative, noninflammatory, and calcification permissive phenotype [100]. After treatment with a hypercholesterolemic diet, the aortic VECs display a protective phenotype described as antiinflammatory, antiapoptotic, and anticalcific [101]. Notably, VECs on the aortic side are more responsive to the diet. More investigation is needed to explain the mechanistic foundations of side-specific VEC phenotypes.

Atherosclerotic swine exhibit a standard human-type inflammatory response in the vasculature [108], so it is notable that the same is not observed in the valve. However, early human CAVD often does not have many inflammatory cells present [11]. In leaflets with mild disease changes, macrophages were only found in $20 \%$ of the lesions and $\mathrm{T}$ cells in 55\% [13], with greater amounts appearing in stenotic valves $[12,13,15]$, ranging from $59 \%$ [15], to $75 \%$ [13] of valves analyzed.

2.3.2. Genetic Porcine Models. Naturally occurring mutations have also been exploited in swine to develop models of nondiet-induced hypercholesterolemia for atherosclerosis research; they may also be suitable as models of CAVD. These models have mutations in the LDLR and/or apolipoprotein genes. Some common models include (1) inherited hyper-low-density lipoprotein and hypercholesterolemia $(\sim 250 \mathrm{mg} / \mathrm{dL})$ from mutant alleles $L p b^{5}, L p r^{1}$, and $L p u^{1}$ for ApoB and ApoU with normal $\operatorname{LDLR}[115,116]$; (2) familial hypercholesterolemia $(130-490 \mathrm{mg} / \mathrm{dL})$ due to an $L D L R$ mutation with altered lipid profiles [117, 118]; or (3) familial hypercholesterolemia due to mutations relating to ApoB and LDLR $(<300 \mathrm{mg} / \mathrm{dL})$ [119]. These models are capable of achieving complex atherosclerotic lesions by two to three years of age without diet induction [115].

2.4. Other Animal Models. Many other animals have been used historically or in certain niche areas as models of atherosclerosis. They include rats, hamsters, pigeons, guinea pigs, cats, dogs, or nonhuman primates and have been reviewed by Moghadasian et al. [88, 107]. Occasionally these animals have been used for CAVD studies [75, 120, 121] and some may be suitable for studying certain comorbidities that directly relate to CAVD $[122,123]$. However, they are not standard models for CAVD currently.

\section{Emerging CAVD Research Themes and the Role of Animal Models}

Increased appreciation and understanding of CAVD as an active, cell-mediated process has renewed interest in valve (patho)biology and the possibility for therapeutic intervention. To this end, priority research areas were recently identified by the National Heart and Lung and Blood Institute Aortic Stenosis Working Group [124] and include (1) improving understanding of the basic biology of CAVD, including signalling pathways and the roles of inflammation and biomechanics in disease initiation and progression; (2) determining the unique contributions of comorbidities to disease development; (3) developing highly sensitive imaging modalities to identify early and subclinical CAVD; (4) determining the feasibility of earlier pharmacological intervention. Research in each of these areas currently benefits from animal models and will continue to require the use of appropriate models in the future, as discussed below.

3.1. Basic Biology of CAVD. The biology of CAVD is complex, involving multiple cellular and molecular regulators, genetic and environmental cues, and interacting signalling cascades [125]. Clearly animal models, notably transgenic mice, are important for dissecting specific signalling pathways and their functional consequences; this topic was recently reviewed [126]. Here we highlight inflammation and biomechanics as two factors that are associated with CAVD and likely influence signalling pathways, but have not been well studied. Animal models are well suited to study inflammation and biomechanics and thus will be critical tools to this end.

3.1.1. Inflammation. Despite the strong association of CAVD with inflammation, the effects of inflammatory cytokines and related factors on valve pathobiology have not been thoroughly studied. Many animal models of CAVD demonstrate inflammation and may therefore be well suited to dissecting the role of inflammation in CAVD pathogenesis. Evidence of inflammation in mice includes significant macrophage [34, 37, 39, 41-43, 46, 47, 59] and T-cell [34, 41] infiltration in some models. Others have seen increased expression of immune response genes [49], MMPs [43, 58], and cathepsins $[42,43,46]$. Lastly, oxidative stress as measured by superoxide production has been exhibited in a number of models [37-40]. In rabbits, macrophages [74, 75, 77-79, 82, 83, 95], foam cells $[75,79]$, T cells [82, 83], and increased MMP-3 expression [103] are reported in valves along with increased hsCRP levels in the blood $[78,85]$. In porcine leaflets, VECs on the disease-prone aortic surface progress from a noninflammatory phenotype in normal valves [100] to an antiinflammatory and antiapoptotic phenotype with early disease [101].

While inflammation has been clearly demonstrated in both animal and human histopathological data, studies are 
typically limited to characterization of the inflammatory response and not the mechanistic causes. Mouse models of CAVD are well suited for studying pathological mechanisms, as conditional knockouts and other genetic manipulations in this species are relatively straightforward. The Ill rn-/mouse exemplifies this approach, as studies with this model have demonstrated a protective role for the interleukin1 receptor antagonist in preventing CAVD onset [60]. However, the lack of a trilayer leaflet morphology in mice differs from that of the human, rabbit, and swine and may impact the relevance of findings in mice to human disease. In addition, care must be taken when employing the common Apoe-/- mouse model to specifically study inflammatory CAVD mechanisms, as recent work has implicated ApoE as a regulator of inflammation [67]. Rabbit and porcine valves have three layers and are large enough to allow study of biomechanical forces and their impact on valvular inflammation. For example, altered fluid flow-induced shear stress induces endothelial expression of VCAM-1, ICAM-1, BMP-4, and TGF- $\beta 1$ [127] and elevated mechanical stretch upregulates MMP and cathepsin activity in porcine leaflets ex vivo [128].

Future animal studies will need to better characterize inflammatory processes at various stages of disease development and begin to dissect the regulatory mechanisms that link inflammation to ECM remodelling and valve dysfunction. The validity and utility of various animal models to study inflammation in CAVD will be further clarified by improved temporal resolution of the pathogenesis of human valvular disease.

3.1.2. Biomechanics. The aortic valve exists in a highly dynamic mechanical environment where it is exposed to significant blood flow-induced shear stresses, pressure loads, flexural deformations, and mechanical resistance from the ECM. Each of these mechanical stimuli regulates valve cell biology and therefore likely contributes to both homeostasis and disease $[129,130]$. However, much of what is known about mechanoregulation of valve biology is based on in vitro or ex vivo studies and the use of normal, nondiseased tissue sources. Animal models are an important, but largely unexploited source of diseased tissue for ex vivo and in vivo studies of valve biomechanics and mechanobiology in CAVD.

To date, the mechanical properties of normal porcine valves have been extensively characterized at multiple length scales: whole leaflets [131-137], individual layers [138, 139], or at higher spatial resolution, focal regions within individual layers [140]. Normal porcine aortic valves have also been used to study the effects of aberrant mechanical forces on valve pathobiology ex vivo [127, 128, 131, 136, 141-143]. The similarity of the trilayer structure, size, and anatomy of the porcine valve to human valve makes them excellent models for structural and biomechanical studies.

To date, the mechanical properties of diseased aortic valve tissue from humans, swine, or other species have not been reported, likely due in part to the limited availability of tissue samples and lack of well-characterized large animal models of CAVD. Such information is critical to defining the role of ECM mechanics in disease regulation [144] and should be a focus of future research. The suitability of porcine valves for biomechanical testing lends promise to the use of porcine CAVD models for the study of biomechanical changes with disease progression. Rabbit models may also be suitable, but their smaller size in comparison to porcine valves makes them less desirable. Mouse valves lack the trilayer humanlike leaflet morphology and are difficult to test mechanically due to their small size ( $\sim 500 \mu \mathrm{m}$ long and $50 \mu \mathrm{m}$ thick) [62]. However, the speed at which advanced disease can be obtained in mice and the feasibility of studying the effect of genetic knockouts on valve mechanics do provide significant benefit. To do this end, micromechanical test methods, like micropipette aspiration (MA), have been adapted recently to characterize mouse leaflet properties [145]. MA also shows promise for its ability to measure focal, microscale tissue properties. Recently, this technique was used to demonstrate that there is significant spatial heterogeneity in the local elastic modulus within individual layers of normal intact porcine aortic valves, but that on average, the fibrosa layer is stiffer than the ventricularis layer, with distinctly stiff and soft regions in the fibrosa and ventricularis, respectively [140]. The use of MA with animal models of CAVD promises to enable high spatial resolution biomechanical testing that is able to address the focal nature of CAVD.

Ultimately, the gap between in vitro/ex vivo biomechanics studies and human disease will need to be bridged by in vivo biomechanical models of CAVD. The valvular mechanical environment and forces experienced by valve cells are defined in part by the external hemodynamic forces that shear and deform the valve leaflets throughout the cardiac cycle. Manipulation of these forces to test direct causal effects of mechanics on valve biology is challenging because of confounding factors that result from invasive manipulation of the valve or perivalvular tissues (e.g., hypertension). As a result, the causal effects of hemodynamic forces on CAVD have yet to be investigated in vivo. Some advances in this area have been made in the study of the effects of hemodynamics on valve development, which were elegantly studied in the zebrafish by using microbeads to impair blood flow [146]. It is believed that aspects of adult valve disease may recapitulate developmental processes [147], and therefore the zebrafish and other developmental models may in fact have some utility for studying the link between hemodynamics and adult valve biology in vivo.

3.2. Contributions of Comorbidities to CAVD. CAVD often coexists with other conditions, including hypercholesterolemia, diabetes mellitus, chronic renal disease, hypertension, metabolic syndrome, and disorders of calcium or phosphate metabolism [148-150]. It is likely that these comorbidities uniquely contribute to the initiation and/or progression of CAVD [124]. A variety of animal models have been used to investigate comorbidities and CAVD, most coupled with a diet that induces hypercholesterolemia to accelerate disease.

A frequently studied comorbidity is familial hypercholesterolemia, an inherited form of hypercholesterolemia that can be caused in humans by more than 800 different mutations [151]. Familial hypercholesterolemic models do 
not require diet induction to induce and accelerate disease, and provide a medium for deeper investigation into specific elements of the disease, especially lipoprotein metabolism. In mice, the Apoe-/- and Ldlr-/-;Apob ${ }^{100}$-only models contain mutations which mimic familial hypercholesterolemia $[38,41]$. As discussed in earlier sections, the WHHL rabbit [84, 93, 104, 105], the St. Thomas Hospital rabbit [94], and a number of porcine models [115-119] also mimic familial hypercholesterolemia. Of these, only the Apoe-/and $L d l r-/-; A p o b^{100}$-only mice models $[38,41]$ and WHHL rabbit [84] have been used to study CAVD to date.

The impact of a variety of other comorbidities on vascular disease has been studied in various species in which hypercholesterolemia was induced by diet. The unique imbalances caused by diseases such as diabetes, chronic renal disease, hypertension, metabolic syndrome, and high serum minerals likely also influence CAVD progression and could lead to necessary unique insights into disease pathways and inciting factors. To date, however, these models have only been applied to study CAVD in mice. For example, the Apoe-/- mouse model has been combined with surgically induced chronic renal disease (CRD) to recapitulate the accelerated CAVD which accompanies human CRD [152]. This 5/6 surgical nephrectomy model with normal diet induces serum hypercholesterolemia after six months $(\sim 400 \mathrm{mg} / \mathrm{dL})$, as well as prototypically high serum phosphate, creatinine, and cystatin $\mathrm{C}$ levels. Valve leaflets stain strongly for microcalcification and macrophages, as detected by near-infrared imaging of targeted nanoparticles [47]. The Apoe-/-;5/6 nephrectomy model has also been combined with the TD.88137 diet and a knockout of the elastolytic proteinase cathepsin S (Ctss-/-). Knockout of cathepsin $S$ reduced valvular calcification, macrophage accumulation, osteogenic and elastolytic activity, and elastin fragmentation [46]. Metabolic syndrome has been recapitulated in WT C57BL/6J mice with the use of a high-fat, high-carbohydrate diet which includes no added cholesterol and produces mild hypercholesterolemia [34], while exercise has been shown to mitigate the effects of the TD.88137 diet in $L d l r-/-$ mice [37]. The development of diabetes is common in many mouse models of aortic valve disease [34, 39], but it is typically a side effect of the studied insult and rarely investigated as a primary initiator of disease [36].

CAVD comorbidities have not been studied in other species, although appropriate putative models do exist. For example, rabbit comorbidity models include diabetes [153], hypertension [154], metabolic syndrome [155, 156], and high serum calcium $[80,82]$, along with hypercholesterolemia. Porcine models have also combined diabetes [106, 108, 110-112] or hypertension [157] with diet-induced hypercholesterolemia. All of these models have been applied to study atherosclerosis, and presumably would be appropriate and effective for studying CAVD, perhaps offering some advantages over murine models.

\subsection{Development of Sensitive Imaging Modalities for Early} CAVD Detection. The ability to arrest or slow CAVD progression will likely require early disease detection, before significant calcium burden and hemodynamic dysfunction have occurred. For this reason, there is significant interest in developing sensitive imaging modalities for early stage detection. Targeted fluorescent nanoparticles are one promising strategy. Commercially available nanoparticles specifically targeting cathepsin $\mathrm{S}$, cathepsin $\mathrm{K}$, cathepsin B, macrophages, VCAM-1, MMP-2/9, and hydroxyapatite have been imaged with intravital dual channel fluorescence imaging in a number of animal studies of CAVD [42, 43, $46,47]$. These or other molecular imaging techniques are promising approaches to detect early human CAVD, and clearly preclinical animal models will be critical to their validation.

Beyond their application in clinical medicine, sensitive noninvasive imaging methods are also important for animal studies, as they enable tracking of disease progression and measurement of cardiac function in vivo over the duration of an experiment. Due to their relatively large size, imaging of rabbits and pigs is typically performed with clinical machines $[80,82,114]$. A possible future trend is to combine nanoparticles with standard imaging modalities for targeted detection. For example, iron oxide (MION-47) and MRI imaging were used to detect early CAVD lesions in rabbits fed a low-cholesterol diet by targeting invading macrophages. However, in these experiments, the MION-47 was also taken up by myofibroblasts in control and cholesterol-fed animals, and therefore more specific targeting may be needed [86].

Many mouse studies continue to employ clinical echocardiography using frequency ranges of $\sim 10-15 \mathrm{MHz}$ to noninvasively examine the functional state of the mouse aortic valve [34, 37-41, 49]. Measurements such as transvalvular blood flow velocity, valve opening time, cusp separation distance, and valve opening area are routinely performed. However, due to the high heart rate of an adult mouse, these frequency ranges only capture 10-20 frames per cardiac cycle [158]. More recently, a high-frequency ultrasound system better suited to imaging the mouse heart has been developed [159]. This system operates at 20-40 MHz, offers a $\sim$ fourfold higher spatial resolution and $\sim$ twofold higher temporal resolution than clinical echo machines [160] and has been validated by magnetic resonance imaging (MRI) as an accurate means to image functional and anatomical parameters of the mouse heart [161]. Increasingly, investigators are taking advantage of this improved technology to delve into the impacts on cardiac function in mouse models of CAVD [50, 53, 59]. Of note, however, is that the higher frequency range of this technique impairs penetration depth and can cause difficulties in successfully imaging aged and obese mice. An alternative is to use high-strength (5-15 T) MRI to image mouse cardiac valves directly $[39,43]$.

3.4. Evaluation of Pharmacological Interventions. Currently, there is no medical treatment for CAVD, and clinical trials have yielded mixed, but generally discouraging, results that motivate the identification and development of new pharmacological interventions to slow or stop the calcific process in CAVD [162]. Clearly, animal models of CAVD are and will continue to be essential to evaluating therapeutics. As is clear from the summary below, the efficacy of various pharmacological interventions depends largely on the stage 
of the disease at the time of drug administration. Thus, translation of results from animal models to humans will be challenging (as CAVD is intentionally accelerated in most models), but must remain a priority.

3.4.1. HMG-CoA Reductase Inhibitors. HMG-CoA reductase inhibitors (statins) were one of the first class of drugs to be directly tested for the treatment of CAVD, with mixed results in both clinical studies [163-166] and NZW rabbit models $[78,83,85]$. In the rabbit studies, statins were administered either concomitant with diet initiation $[78,85]$ or 15 months after diet initiation, by which point there was established sclerosis but no calcification or functional abnormalities [83]. In the former case, eight weeks of simultaneous atherogenic diet and statin treatment resulted in decreased total cholesterol, leaflet thickness, OPN, macrophage infiltration, cell proliferation, and Runx2 expression compared with diet-only samples [78]. However, the high-cholesterol diet used in this study induced lesions throughout the leaflet, which does not mimic the fibrosa specificity of human disease. In a subsequent study using a lower cholesterol diet, three months of statin treatment (initiated at the same time as dietary insult) decreased the amount of calcification and increased eNOS expression [85]. In WHHL rabbits, atorvastatin attenuated hypercholesterolemia-induced calcification when administered concomitantly with diet, in part through the LDLR LRP5/ $\beta$-catenin pathway [84]. In contrast, when statins were administered for 15 months after disease had already been established in NZW rabbits, treatment decreased inflammatory cell infiltration and OPN expression but did not prevent calcification or collagen deposition, reduce lipid burden, or prevent the functional impairment that occurred without statin treatment [83].

Statins have not been extensively tested in mouse models of CAVD, though preliminary studies with pitavastatin in Ill $r n-/$ - mice are reported to have no therapeutic benefit [60]. However, aggressive lipid lowering has been more directly achieved with the Reversa mouse model, which significantly normalizes plasma cholesterol levels with the use of a genetic switch $(\sim 800 \rightarrow \sim 200 \mathrm{mg} / \mathrm{dL})[39,40]$. Early intervention after six months on the TD.88137 diet was able to reverse nearly all pathological signs of CAVD, but reversal at 12 months after significant calcium burden had already developed did not produce measureable improvements in aortic valve function.

In total, preclinical and clinical trials to date do not support statin therapy as a primary treatment for patients with valvular heart disease to slow its progression [124]. The inconsistent results among the clinical trials likely reflect many differences, including enrolment criteria and timing of therapy [124], which are reflected in the animal studies by the extent of disease at the time of treatment. Thus, it is important that animal models mimic the progression and severity of human disease and experimental designs match the clinical situation as closely as possible, so that preclinical data can be translated to inform the design and interpretation of future clinical trials of statins or other pharmacological interventions.
3.4.2. Renin-Angiotensin-Aldosterone System Inhibitors. The renin-angiotensin-aldosterone system (RAAS) controls blood pressure and fluid and electrolyte balances, and members of this family may have roles in normal physiological repair of the valve. Dysregulation of RAAS components can have proatherothrombotic effects resulting in pathologic fibrosis and calcification of the aortic valve [167]. The major members of this family implicated in CAVD are ACE, AngII, and AT1R [20, 23]. Aldosterone receptors have also been shown to be present in rabbit aortic valves [96]. AngII, produced by ACE and mediated by the AT1R, may contribute to CAVD by promoting macrophage lipid accumulation and inflammation, increasing oxidative stress, impairing fibrinolysis, and stimulating production of the proteoglycan biglycan, which can retain lipoproteins [125].

ACE inhibitors, angiotensin receptor blockers (ARBs), and aldosterone receptor antagonists (ARAs) have begun to be investigated for treatment of CAVD. ACE inhibitors have shown mixed results in retrospective clinical studies with one showing a strong association between the use of ACE inhibitors and decreased valve calcification [168] and the other finding no effects [169]. In NZWR, treatment with the ACE inhibitor ramipril concomitantly with induction of CAVD by VitD2 at 25000 IU (four days per week) for eight weeks retarded the development of CAVD [99]. The ARB olmesartan decreased macrophage and myofibroblast accumulation, decreased $O P N, A C E$, and Runx2 mRNA expression, and maintained endothelial integrity when administered to NZWR during the last four weeks of an eight-week 1\% cholesterol diet treatment [95]. The ARA eplerenone showed no effect in clinical trials of moderate- to severe stenosis [170]. However, administration of eplerenone to NZWR for the last four weeks of an eight-week $1 \%$ cholesterol diet elevated aldosterone levels without altering blood pressure and decreased macrophage accumulation, ACE expression, and calcification within the leaflets [96].

3.4.3. Antioxidants. Reactive oxygen species are present in both human [20, 23] and rabbit [19] valves during CAVD. The antioxidative compounds tempol and lipoic acid (LA) were tested individually by concomitant administration with a CAVD-inducing diet of $0.5 \%$ cholesterol and VitD2 in NZWR. Tempol decreased superoxide presence but led to an increase in hydrogen peroxide, $\mathrm{NAD}(\mathrm{P}) \mathrm{H}$ oxidase subunits, and calcification. LA decreased both superoxide and hydrogen peroxide and led to a decrease in calcification and echogenicity of the valve. These preliminary results showed that ROS may potentiate calcification, particularly in relation to hydrogen peroxide and possibly $\mathrm{NAD}(\mathrm{P}) \mathrm{H}$ oxidase activity, and pharmacological prevention of ROS may prevent or decrease calcific burden [19].

\section{Conclusions and Recommendations}

When properly chosen and implemented, animal models are a vital tool for the investigation of pathobiological mechanisms of CAVD and potential therapeutic interventions. Many animal models have been shown to recapitulate 
important aspects of human CAVD pathobiology, thus enabling detailed investigations that are otherwise unfeasible or impossible to conduct in humans. The development of new models and our improved understanding of the utility of existing models have quickly advanced the impact that animal model-based studies are making within this field. Mouse, rabbit, or swine models each offer speciesspecific advantages for the study of CAVD and represent a fundamental step forward in the translation of basic scientific research into clinically relevant and impactful knowledge.

Moving forward, there is a need for improved model characterization to enable direct comparisons between multiple studies and aid in interpretation of findings as they related to human CAVD. At a minimum, the following model parameters necessary for interstudy comparisons should be provided: (1) animal starting age and weight; (2) full diet composition, including \% kcal contributions; (3) feeding regime (ad libitum, weight adjusted, or fixed amount, and growth rate); (4) full plasma lipid profiles (TC, HDL, TG, LDL); (5) background strain(s) used in generating knockout mice; (6) age and weight at sacrifice. Models should have full histological characterization of lesion progression, including lesion composition and anatomical location, and valvular function should be impaired in advanced disease models. Some mice and rabbit models acquire hemodynamically significant CAVD, but the same has yet to be shown in swine. However, some swine models develop advanced atherosclerotic lesions and vascular stiffening after eight months of high-cholesterol diet [112], and therefore valvular stiffening and dysfunction may simply take longer than has been studied to date [101].

In order to truly understand the relevance of animal models to human valvular disease, a better understanding of human CAVD pathogenesis is required. As new insights into human disease are revealed, animal models and the data they generate need to be critically reinterpreted. A priority is to determine whether the extreme hypercholesterolemia induced in many standard CAVD models faithfully represents the full progression of human disease. In the future, ageing models of disease may prove to be the best at inducing human-type disease, although time and expense remain prohibitive. To date, there are two aged mouse models [38, 41] and one aged rabbit model [83] that maintain moderate cholesterol levels and induce functional disease. Swine models of familial or mild diet-induced hypercholesterolemia could provide the best match with cholesterol levels in humans and when aged, may produce advanced disease.

Perhaps the most promising application of well-characterized animal models is to investigate CAVD-initiating events and the mechanisms of its progression to late-stage functional impairment. Animal models clearly offer a significant advantage in this context, as it is difficult to clearly identify disease stage or control for confounding factors in human autopsy or transplant samples. CAVD initiation and early progression have largely been ignored to date, but would provide the richest insight into therapeutic targets to arrest calcification before the putative "point of no return" when calcific burden cannot be reversed. Inflammation, ECM adaptation, and early procalcific signalling likely play important roles, but have not yet been studied in detail. There has been little focus on, for example, the mechanisms that regulate macrophage/T-cell infiltration, inflammatory signalling cascades, or layer-specific ECM changes and their role in modulating VIC phenotype. It is prospective investigations of molecular regulators of valve homeostasis and disease progression like these that will benefit from wellcharacterized and validated animal models and are most likely to lead to the discovery of novel treatments for CAVD.

\section{Author's Contribution}

Krista L. Sider and Mark C. Blaster contributed equally to this paper.

\section{References}

[1] V. L. Roger, A. S. Go, D. M. Lloyd-Jones et al., "Heart disease and stroke statistics—2011 update: report from the American Heart Association," Circulation, vol. 123, no. 4, pp. e18-e209, 2011.

[2] E. Rabkin-Aikawa, M. Farber, M. Aikawa, and F. J. Schoen, "Dynamic and reversible changes of interstitial cell phenotype during remodeling of cardiac valves," The Journal of Heart Valve Disease, vol. 13, no. 5, pp. 841-847, 2004.

[3] A. C. Liu, V. R. Joag, and A. I. Gotlieb, “The emerging role of valve interstitial cell phenotypes in regulating heart valve pathobiology," The American Journal of Pathology, vol. 171, no. 5, pp. 1407-1418, 2007.

[4] J. H. Chen, C. Y. Yip, E. D. Sone, and C. A. Simmons, "Identification and characterization of aortic valve mesenchymal progenitor cells with robust osteogenic calcification potential," The American Journal of Pathology, vol. 174, no. 3, pp. 1109-1119, 2009.

[5] F. J. Schoen, "Evolving concepts of cardiac valve dynamics: the continuum of development, functional structure, pathobiology, and tissue engineering," Circulation, vol. 118, no. 18, pp. 1864-1880, 2008.

[6] C. M. Otto, B. K. Lind, D. W. Kitzman, B. J. Gersh, and D. S. Siscovick, "Association of aortic-valve sclerosis with cardiovascular mortality and morbidity in the elderly," The New England Journal of Medicine, vol. 341, no. 3, pp. 142147, 1999.

[7] I. Ben-Dor, A. D. Pichard, M. A. Gonzalez et al., "Correlates and causes of death in patients with severe symptomatic aortic stenosis who are not eligible to participate in a clinical trial of transcatheter aortic valve implantation," Circulation, vol. 122, no. 11, supplement, pp. S37-S42, 2010.

[8] B. F. Stewart, D. Siscovick, B. K. Lind et al., "Clinical factors associated with calcific aortic valve disease. Cardiovascular health study," The Journal of American College of Cardiology, vol. 29, no. 3, pp. 630-634, 1997.

[9] A. Boon, E. Cheriex, J. Lodder, and F. Kessels, "Cardiac valve calcification: characteristics of patients with calcification of the mitral annulus or aortic valve," Heart, vol. 78, no. 5, pp. 472-474, 1997.

[10] J. H. Ix, M. G. Shlipak, R. Katz et al., "Kidney function and aortic valve and mitral annular calcification in the multi-ethnic study of atherosclerosis (MESA)," The American Journal of Kidney Diseases, vol. 50, no. 3, pp. 412-420, 2007. 
[11] K. D. O’Brien, D. D. Reichenbach, S. M. Marcovina, J. Kuusisto, C. E. Alpers, and C. M. Otto, "Apolipoproteins B, (a), and $\mathrm{E}$ accumulate in the morphologically early lesion of 'degenerative' valvular aortic stenosis," Arteriosclerosis, Thrombosis, and Vascular Biology, vol. 16, no. 4, pp. 523-532, 1996.

[12] M. Olsson, J. Thyberg, and J. Nilsson, "Presence of oxidized low density lipoprotein in nonrheumatic stenotic aortic valves," Arteriosclerosis, Thrombosis, and Vascular Biology, vol. 19, no. 5, pp. 1218-1222, 1999.

[13] C. M. Otto, J. Kuusisto, D. D. Reichenbach, A. M. Gown, and K. D. O'Brien, "Characterization of the early lesion of 'degenerative' valvular aortic stenosis: histological and immunohistochemical studies," Circulation, vol. 90, no. 2, pp. 844-853, 1994.

[14] J. J. Kaden, C. E. Dempfle, R. Grobholz et al., "Inflammatory regulation of extracellular matrix remodeling in calcific aortic valve stenosis," Cardiovascular Pathology, vol. 14, no. 2, pp. 80-87, 2005.

[15] B. A. Warren and J. L. Yong, "Calcification of the aortic valve: its progression and grading," Pathology, vol. 29, no. 4, pp. 360-368, 1997.

[16] S. Helske, R. Oksjoki, K. A. Lindstedt et al., "Complement system is activated in stenotic aortic valves," Atherosclerosis, vol. 196, no. 1, pp. 190-200, 2008.

[17] E. R. Mohler, F. Gannon, C. Reynolds, R. Zimmerman, M. G. Keane, and F. S. Kaplan, "Bone formation and inflammation in cardiac valves," Circulation, vol. 103, no. 11, pp. 1522$1528,2001$.

[18] J. D. Miller, Y. Chu, R. M. Brooks, W. E. Richenbacher, R. Peña-Silva, and D. D. Heistad, "Dysregulation of antioxidant mechanisms contributes to increased oxidative stress in calcific aortic valvular stenosis in humans," Journal of the American College of Cardiology, vol. 52, no. 10, pp. 843-850, 2008.

[19] M. Liberman, E. Bassi, M. K. Martinatti et al., "Oxidant generation predominates around calcifying foci and enhances progression of aortic valve calcification," Arteriosclerosis, Thrombosis, and Vascular Biology, vol. 28, no. 3, pp. 463-470, 2008.

[20] S. Helske, K. A. Lindstedt, M. Laine et al., "Induction of local angiotensin II-producing systems in stenotic aortic valves," Journal of the American College of Cardiology, vol. 44, no. 9, pp. 1859-1866, 2004.

[21] M. E. Edep, J. Shirani, P. Wolf, and D. L. Brown, "Matrix metalloproteinase expression in nonrheumatic aortic stenosis," Cardiovascular Pathology, vol. 9, no. 5, pp. 281-286, 2000.

[22] J. J. Kaden, C. E. Dempfle, R. Grobholz et al., "Interleukin-1 beta promotes matrix metalloproteinase expression and cell proliferation in calcific aortic valve stenosis," Atherosclerosis, vol. 170, no. 2, pp. 205-211, 2003.

[23] K. D. O’Brien, D. M. Shavelle, M. T. Caulfield et al., "Association of angiotensin-converting enzyme with low-density lipoprotein in aortic valvular lesions and in human plasma," Circulation, vol. 106, no. 17, pp. 2224-2230, 2002.

[24] A. Mazzone, M. C. Epistolato, R. De Caterina et al., "Neoangiogenesis, T-lymphocyte infiltration, and heat shock protein-60 are biological hallmarks of an immunomediated inflammatory process in end-stage calcified aortic valve stenosis," Journal of the American College of Cardiology, vol. 43, no. 9, pp. 1670-1676, 2004.

[25] N. K. Ghaisas, J. B. Foley, D. S. O’Briain, P. Crean, D. Kelleher, and M. Walsh, "Adhesion molecules in nonrheumatic aortic valve disease: endothelial expression, serum levels and effects of valve replacement," Journal of the American College of Cardiology, vol. 36, no. 7, pp. 2257-2262, 2000.

[26] H. A. Eriksen, J. Satta, J. Risteli, M. Veijola, P. Väre, and Y. Soini, "Type I and type III collagen synthesis and composition in the valve matrix in aortic valve stenosis," Atherosclerosis, vol. 189, no. 1, pp. 91-98, 2006.

[27] R. B. Hinton, J. Lincoln, G. H. Deutsch et al., "Extracellular matrix remodeling and organization in developing and diseased aortic valves," Circulation Research, vol. 98, no. 11, pp. 1431-1438, 2006.

[28] E. H. Stephens, J. G. Saltarrelli, and L. S. Baggett, "Differential proteoglycan and hyaluronan distribution in calcified aortic valves," Cardiovascular Pathology. In press.

[29] O. Fondard, D. Detaint, B. Iung et al., "Extracellular matrix remodelling in human aortic valve disease: the role of matrix metalloproteinases and their tissue inhibitors," European Heart Journal, vol. 26, no. 13, pp. 1333-1341, 2005.

[30] J. Satta, J. Oiva, T. Salo et al., "Evidence for an altered balance between matrix metalloproteinase- 9 and its inhibitors in calcific aortic stenosis," Annals of Thoracic Surgery, vol. 76, no. 3, pp. 681-688, 2003.

[31] A. H. Rapp, L. D. Hillis, R. A. Lange, and J. E. Cigarroa, "Prevalence of coronary artery disease in patients with aortic stenosis with and without angina pectoris," American Journal of Cardiology, vol. 87, no. 10, pp. 1216-1217, 2001.

[32] M. Guerraty and E. R. Mohler, "Models of aortic valve calcification," Journal of Investigative Medicine, vol. 55, no. 6, pp. 278-283, 2007.

[33] S. Fazio and M. F. Linton, "Mouse models of hyperlipidemia and atherosclerosis," Frontiers in Bioscience, vol. 6, pp. D515D525, 2001.

[34] M. C. Drolet, E. Roussel, Y. Deshaies, J. Couet, and M. Arsenault, "A high fat/high carbohydrate diet induces aortic valve disease in C57BL/6J mice," The Journal of the American College of Cardiology, vol. 47, no. 4, pp. 850-855, 2006.

[35] M. Mehrabian, L. L. Demer, and A. J. Lusis, "Differential accumulation of intimal monocyte-macrophages relative to lipoproteins and lipofuscin corresponds to hemodynamic forces on cardiac valves in mice," Arteriosclerosis and Thrombosis, vol. 11, no. 4, pp. 947-957, 1991.

[36] D. A. Towler, M. Bidder, T. Latifi, T. Coleman, and C. F. Semenkovich, "Diet-induced diabetes activates an osteogenic gene regulatory program in the aortas of low density lipoprotein receptor-deficient mice," The Journal of Biological Chemistry, vol. 273, no. 46, pp. 30427-30434, 1998.

[37] Y. Matsumoto, V. Adams, S. Jacob, N. Mangner, G. Schuler, and A. Linke, "Regular exercise training prevents aortic valve disease in low-density lipoprotein-receptor-deficient mice," Circulation, vol. 121, no. 6, pp. 759-767, 2010.

[38] R. M. Weiss, M. Ohashi, J. D. Miller, S. G. Young, and D. D. Heistad, "Calcific aortic valve stenosis in old hypercholesterolemic mice," Circulation, vol. 114, no. 19, pp. 2065-2069, 2006.

[39] J. D. Miller, R. M. Weiss, K. M. Serrano et al., "Lowering plasma cholesterol levels halts progression of aortic valve disease in mice," Circulation, vol. 119, no. 20, pp. 2693-2701, 2009.

[40] J. D. Miller, R. M. Weiss, K. M. Serrano et al., "Evidence for active regulation of pro-osteogenic signaling in advanced aortic valve disease," Arteriosclerosis, Thrombosis, and Vascular Biology, vol. 30, no. 12, pp. 2482-2486, 2010.

[41] K. Tanaka, M. Sata, D. Fukuda et al., "Age-associated aortic stenosis in apolipoprotein E-deficient mice," The Journal of 
the American College of Cardiology, vol. 46, no. 1, pp. 134141, 2005.

[42] E. Aikawa, M. Nahrendorf, J. L. Figueiredo et al., "Osteogenesis associates with inflammation in early-stage atherosclerosis evaluated by molecular imaging in vivo," Circulation, vol. 116, no. 24, pp. 2841-2850, 2007.

[43] E. Aikawa, M. Nahrendorf, D. Sosnovik et al., "Multimodality molecular imaging identifies proteolytic and osteogenic activities in early aortic valve disease," Circulation, vol. 115, no. 3, pp. 377-386, 2007.

[44] M. Zeadin, M. Butcher, G. Werstuck, M. Khan, C. K. Yee, and S. G. Shaughnessy, "Effect of leptin on vascular calcification in apolipoprotein E-deficient mice," Arteriosclerosis, Thrombosis, and Vascular Biology, vol. 29, no. 12, pp. 2069-2075, 2009.

[45] S. Srivastava, S. D. Sithu, E. Vladykovskaya et al., "Oral exposure to acrolein exacerbates atherosclerosis in apoE-null mice," Atherosclerosis, vol. 215, no. 2, pp. 301-308, 2011.

[46] E. Aikawa, M. Aikawa, G. Rusanescu et al., "Arterial and aortic valve calcification abolished by elastolytic cathepsin $S$ deficiency in chronic renal disease," Circulation, vol. 119, no. 13, pp. 1785-1794, 2009.

[47] J. Hjortnaes, J. Butcher, J. L. Figueiredo et al., "Arterial and aortic valve calcification inversely correlates with osteoporotic bone remodelling: a role for inflammation," The European Heart Journal, vol. 31, no. 16, pp. 1975-1984, 2010.

[48] G. Luo, P. Ducy, M. D. McKee et al., "Spontaneous calcification of arteries and cartilage in mice lacking matrix GLA protien," Nature, vol. 386, no. 6620, pp. 78-81, 1997.

[49] K. Hanada, M. Vermeij, G. A. Garinis et al., "Perturbations of vascular homeostasis and aortic valve abnormalities in fibulin-4 deficient mice," Circulation Research, vol. 100, no. 5, pp. 738-746, 2007.

[50] R. B. Hinton, J. Adelman-Brown, S. Witt et al., "Elastin haploinsufficiency results in progressive aortic valve malformation and latent valve disease in a mouse model," Circulation Research, vol. 107, no. 4, pp. 549-557, 2010.

[51] V. Nigam and D. Srivastava, "Notch1 represses osteogenic pathways in aortic valve cells," Journal of Molecular and Cellular Cardiology, vol. 47, no. 6, pp. 828-834, 2009.

[52] M. Nus, D. MacGrogan, B. Martínez-Poveda et al., "Dietinduced aortic valve disease in mice haploinsufficient for the notch pathway effector RBPJK/CSL," Arteriosclerosis, Thrombosis, and Vascular Biology, vol. 31, no. 7, pp. 1580-1588, 2011.

[53] T. V. Tkatchenko, R. A. Moreno-Rodriguez, S. J. Conway, J. D. Molkentin, R. R. Markwald, and A. V. Tkatchenko, "Lack of periostin leads to suppression of Notch1 signaling and calcific aortic valve disease," Physiological Genomics, vol. 39, no. 3, pp. 160-168, 2009.

[54] D. Hakuno, N. Kimura, M. Yoshioka et al., "Periostin advances atherosclerotic and rheumatic cardiac valve degeneration by inducing angiogenesis and MMP production in humans and rodents," The Journal of Clinical Investigation, vol. 120, no. 7, pp. 2292-2306, 2010.

[55] M. Yoshioka, S. Yuasa, K. Matsumura et al., "Chondromodulin-I maintains cardiac valvular function by preventing angiogenesis," Nature Medicine, vol. 12, no. 10, pp. 11511159, 2006.

[56] T. C. Lee, Y. D. Zhao, D. W. Courtman, and D. J. Stewart, "Abnormal aortic valve development in mice lacking endothelial nitric oxide synthase," Circulation, vol. 101, no. 20, pp. 2345-2348, 2000.
[57] K. M. Galvin, M. J. Donovan, C. A. Lynch et al., "A role for Smad6 in development and homeostasis of the cardiovascular system," Nature Genetics, vol. 24, no. 2, pp. 171-174, 2000.

[58] S. Chakraborty, E. E. Wirrig, R. B. Hinton, W. H. Merrill, D. B. Spicer, and K. E. Yutzey, "Twist1 promotes heart valve cell proliferation and extracellular matrix gene expression during development in vivo and is expressed in human diseased aortic valves," Developmental Biology, vol. 347, no. 1, pp. 167$179,2010$.

[59] C. J. Barrick, R. B. Roberts, M. Rojas et al., "Reduced EGFR causes abnormal valvular differentiation leading to calcific aortic stenosis and left ventricular hypertrophy in C57BL/6J but not 129S1/SvImJ mice," American Journal of Physiology, vol. 297, no. 1, pp. H65-H75, 2009.

[60] K. Isoda, T. Matsuki, H. Kondo, Y. Iwakura, and F. Ohsuzu, "Deficiency of interleukin-1 receptor antagonist induces aortic valve disease in BALB/c Mice," Arteriosclerosis, Thrombosis, and Vascular Biology, vol. 30, no. 4, pp. 708-715, 2010.

[61] D. W. Swinkels and P. N. M. Demacker, "Comparative studies on the low density lipoprotein subfractions from pig and man," Comparative Biochemistry and Physiology, vol. 90, no. 2, pp. 297-300, 1988.

[62] R. B. Hinton Jr., C. M. Alfieri, S. A. Witt et al., "Mouse heart valve structure and function: echocardiographic and morphometric analyses from the fetus through the aged adult," The American Journal of Physiology, vol. 294, no. 6, pp. H2480-H2488, 2008.

[63] A. Daugherty and D. L. Rateri, "Development of experimental designs for atherosclerosis studies in mice," Methods, vol. 36, no. 2, pp. 129-138, 2005.

[64] H. M. Dansky, S. A. Charlton, J. L. Sikes et al., "Genetic background determines the extent of atherosclerosis in ApoEdeficient mice," Arteriosclerosis, Thrombosis, and Vascular Biology, vol. 19, no. 8, pp. 1960-1968, 1999.

[65] W. Shi, N. J. Wang, D. M. Shih, V. Z. Sun, X. Wang, and A. J. Lusis, "Determinants of atherosclerosis susceptibility in the $\mathrm{C} 3 \mathrm{H}$ and $\mathrm{C} 57 \mathrm{BL} / 6$ mouse model: evidence for involvement of endothelial cells but not blood cells or cholesterol metabolism," Circulation Research, vol. 86, no. 10, pp. 10781084, 2000.

[66] C. H. Mjaatvedt, C. B. Kern, R. A. Norris, S. Fairey, and C. L. Cave, "Normal distribution of melanocytes in the mouse heart," Anatomical Record-Part A, vol. 285, no. 2, pp. 748757, 2005.

[67] H. L. Zhang, J. Wu, and J. Zhu, "The role of apolipoprotein E in Guillain-Barré syndrome and experimental autoimmune neuritis," Journal of Biomedicine and Biotechnology, vol. 2010, Article ID 357412, 2010.

[68] A. S. Plump, J. D. Smith, T. Hayek et al., "Severe hypercholesterolemia and atherosclerosis in apolipoprotein E- deficient mice created by homologous recombination in ES cells," Cell, vol. 71, no. 2, pp. 343-353, 1992.

[69] J. I. E. Hoffman and S. Kaplan, "The incidence of congenital heart disease," Journal of the American College of Cardiology, vol. 39, no. 12, pp. 1890-1900, 2002.

[70] V. Garg, A. N. Muth, J. F. Ransom et al., "Mutations in NOTCH1 cause aortic valve disease," Nature, vol. 437, no. 7056, pp. 270-274, 2005.

[71] D. Aicher, C. Urbich, A. Zeiher, S. Dimmeler, and H. J. Schäfers, "Endothelial nitric oxide synthase in bicuspid aortic valve disease," Annals of Thoracic Surgery, vol. 83, no. 4, pp. 1290-1294, 2007.

[72] W. Shi, X. Wang, D. M. Shih, V. E. Laubach, M. Navab, and A. J. Lusis, "Paradoxical reduction of fatty streak formation in 
mice lacking endothelial nitric oxide synthase," Circulation, vol. 105, no. 17, pp. 2078-2082, 2002.

[73] N. M. Rajamannan, "Bicuspid aortic valve disease: the role of oxidative stress in Lrp5 bone formation," Cardiovascular Pathology, vol. 20, no. 3, pp. 168-176, 2011.

[74] M. Cimini, D. R. Boughner, J. A. Ronald, L. Aldington, and K. A. Rogers, "Development of aortic valve sclerosis in a rabbit model of atherosclerosis: an immunohistochemical and histological study," Journal of Heart Valve Disease, vol. 14, no. 3, pp. 365-375, 2005.

[75] D. A. Filip, A. Nistor, and A. Bulla, "Cellular events in the development of valvular atherosclerotic lesions induced by experimental hypercholesterolemia," Atherosclerosis, vol. 67, no. 2-3, pp. 199-214, 1987.

[76] P. Nievelstein-Post, G. Mottino, A. Fogelman, and J. Frank, "An ultrastructural study of lipoprotein accumulation in cardiac valves of the rabbit," Arteriosclerosis and Thrombosis, vol. 14, no. 7, pp. 1151-1161, 1994.

[77] M. E. Haberland, G. Mottino, M. Le, and J. S. Frank, "Sequestration of aggregated LDL by macrophages studied with freeze-etch electron microscopy," Journal of Lipid Research, vol. 42, no. 4, pp. 605-619, 2001.

[78] N. M. Rajamannan, M. Subramaniam, M. Springett et al., "Atorvastatin inhibits hypercholesterolemia-induced cellular proliferation and bone matrix production in the rabbit aortic valve," Circulation, vol. 105, no. 22, pp. 2660-2665, 2002.

[79] N. M. Rajamannan, G. Sangiorgi, M. Springett et al., "Experimental hypercholesterolemia induces apoptosis in the aortic valve," Journal of Heart Valve Disease, vol. 10, no. 3, pp. 371374, 2001.

[80] M. C. Drolet, M. Arsenault, and J. Couet, "Experimental aortic valve stenosis in rabbits," Journal of the American College of Cardiology, vol. 41, no. 7, pp. 1211-1217, 2003.

[81] Z. Zeng, P. Nievelstein-Post, Y. Yin, K. M. Jan, J. S. Frank, and D. S. Rumschitzki, "Macromolecular transport in heart valves. III. Experiment and theory for the size distribution of extracellular liposomes in hyperlipidemic rabbits," The American Journal of Physiology, vol. 292, no. 6, pp. H2687H2697, 2007.

[82] M. C. Drolet, J. Couet, and M. Arsenault, “Development of aortic valve sclerosis or stenosis in rabbits: role of cholesterol and calcium," Journal of Heart Valve Disease, vol. 17, no. 4, pp. 381-387, 2008.

[83] A. M. Hamilton, D. R. Boughner, M. Drangova, and K. A. Rogers, "Statin treatment of hypercholesterolemic-induced aortic valve sclerosis," Cardiovascular Pathology, vol. 20, no. 2, pp. 84-92, 2010.

[84] N. M. Rajamannan, M. Subramaniam, F. Caira, S. R. Stock, and T. C. Spelsberg, "Atorvastatin inhibits hypercholesterolemia-induced calcification in the aortic valves via the Lrp5 receptor pathway," Circulation, vol. 112, no. 9, supplement, pp. I229-I234, 2005.

[85] N. M. Rajamannan, M. Subramaniam, S. R. Stock et al., "Atorvastatin inhibits calcification and enhances nitric oxide synthase production in the hypercholesterolaemic aortic valve," Heart, vol. 91, no. 6, pp. 806-810, 2005.

[86] A. M. Hamilton, K. A. Rogers, A. J. L. Belisle et al., "Early identification of aortic valve sclerosis using iron oxide enhanced MRI," Journal of Magnetic Resonance Imaging, vol. 31, no. 1, pp. 110-116, 2010.

[87] M. E. Brousseau and J. M. Hoeg, "Transgenic rabbits as models for atherosclerosis research," Journal of Lipid Research, vol. 40, no. 3, pp. 365-375, 1999.
[88] M. H. Moghadasian, J. J. Frohlich, and B. M. McManus, "Advances in experimental dyslipidemia and atherosclerosis," Laboratory Investigation, vol. 81, no. 9, pp. 1173-1183, 2001.

[89] J. R. Turk and M. H. Laughlin, "Physical activity and atherosclerosis: which animal model?" Canadian Journal of Applied Physiology, vol. 29, no. 5, pp. 657-683, 2004.

[90] J. Fan, S. P. A. McCormick, R. M. Krauss et al., "Overexpression of human apolipoprotein B-100 in transgenic rabbits results in increased levels of LDL and decreased levels of HDL," Arteriosclerosis, Thrombosis, and Vascular Biology, vol. 15, no. 11, pp. 1889-1899, 1995.

[91] J. Fan, H. Shimoyamada, H. Sun, S. Marcovina, K. Honda, and T. Watanabe, "Transgenic rabbits expressing human apolipoprotein(a) develop more extensive atherosclerotic lesions in response to a cholesterol-rich diet," Arteriosclerosis, Thrombosis, and Vascular Biology, vol. 21, no. 1, pp. 88-94, 2001.

[92] J. Fan and T. Watanabe, "Cholesterol-fed and transgenic rabbit models for the study of atherosclerosis," Journal of Atherosclerosis and Thrombosis, vol. 7, no. 1, pp. 26-32, 2000.

[93] G. Aliev and G. Burnstock, "Watanabe rabbits with heritable hypercholesterolaemia: a model of atherosclerosis," Histology and Histopathology, vol. 13, no. 3, pp. 797-817, 1998.

[94] T. H. Beaty, V. L. Prenger, D. G. Virgil, B. Lewis, P. O. Kwiterovich, and P. S. Bachorik, "A genetic model for control of hypertriglyceridemia and apolipoprotein B levels in the Johns Hopkins colony of St. Thomas Hospital rabbits," Genetics, vol. 132, no. 4, pp. 1095-1104, 1992.

[95] K. Arishiro, M. Hoshiga, N. Negoro et al., "Angiotensin receptor-1 blocker inhibits atherosclerotic changes and endothelial disruption of the aortic valve in hypercholesterolemic rabbits," Journal of the American College of Cardiology, vol. 49, no. 13, pp. 1482-1489, 2007.

[96] S. Gkizas, D. Koumoundourou, X. Sirinian et al., "Aldosterone receptor blockade inhibits degenerative processes in the early stage of calcific aortic stenosis," European Journal of Pharmacology, vol. 642, no. 1-3, pp. 107-112, 2010.

[97] S. Marechaux, D. Corseaux, A. Vincentelli et al., "Identification of tissue factor in experimental aortic valve sclerosis," Cardiovascular Pathology, vol. 18, no. 2, pp. 67-76, 2009.

[98] D. T. M. Ngo, I. Stafford, D. J. Kelly et al., "Vitamin D supplementation induces the development of aortic stenosis in rabbits: interactions with endothelial function and thioredoxininteracting protein," European Journal of Pharmacology, vol. 590, no. $1-3$, pp. 290-296, 2008.

[99] D. T. Ngo, I. Stafford, A. L. Sverdlov et al., "Ramipril retards development of aortic valve stenosis in a rabbit model: mechanistic considerations," British Journal of Pharmacology, vol. 162, no. 3, pp. 722-732, 2011.

[100] C. A. Simmons, G. R. Grant, E. Manduchi, and P. F. Davies, "Spatial heterogeneity of endothelial phenotypes correlates with side-specific vulnerability to calcification in normal porcine aortic valves," Circulation Research, vol. 96, no. 7, pp. 792-799, 2005.

[101] M. A. Guerraty, G. R. Grant, J. W. Karanian, O. A. Chiesa, W. F. Pritchard, and P. F. Davies, "Hypercholesterolemia induces side-specific phenotypic changes and peroxisome proliferator-activated receptor- gamma pathway activation in swine aortic valve endothelium," Arteriosclerosis, Thrombosis, and Vascular Biology, vol. 30, no. 2, pp. 225-231, 2010.

[102] J. T. Prior, D. M. Kurtz, and D. D. Ziegler, "The hypercholesteremic rabbit. An aid to understanding arteriosclerosis in man?” Archives of Pathology, vol. 71, pp. 672-684, 1961. 
[103] H. M. Kwon, B. K. Lee, D. Kim et al., "Experimental hypercholesterolemia induces ultrastructural changes in the elastic laminae of rabbit aortic valve," Yonsei Medical Journal, vol. 39, no. 4, pp. 345-354, 1998.

[104] M. E. Brousseau, R. D. Kauffman, E. E. Herderick et al., "LCAT modulates atherogenic plasma lipoproteins and the extent of atherosclerosis only in the presence of normal LDL receptors in transgenic rabbits," Arteriosclerosis, Thrombosis, and Vascular Biology, vol. 20, no. 2, pp. 450-458, 2000.

[105] T. Kazuhiko, I. Yasuhide, and M. Toshio, "Effects of NO-1886, a lipoprotein lipase promoting agent, on homozygous and heterozygous Watanabe heritable hyperlipidaemic rabbits," Arzneimittel-Forschung, vol. 50, no. 2, pp. 118-121, 2000.

[106] J. L. Dixon, J. D. Stoops, J. L. Parker, M. H. Laughlin, G. A. Weisman, and M. Sturek, "Dyslipidemia and vascular dysfunction in diabetic pigs fed an atherogenic diet," Arteriosclerosis, Thrombosis, and Vascular Biology, vol. 19, no. 12, pp. 2981-2992, 1999.

[107] M. H. Moghadasian, "Experimental atherosclerosis: a historical overview," Life Sciences, vol. 70, no. 8, pp. 855-865, 2002.

[108] R. G. Gerrity, R. Natarajan, J. L. Nadler, and T. Kimsey, "Diabetes-induced accelerated atherosclerosis in swine," Diabetes, vol. 50, no. 7, pp. 1654-1665, 2001.

[109] R. G. Gerrity, H. K. Naito, M. Richardson, and C. J. Schwartz, "Dietary induced atherogenesis in swine. Morphology of the intima in prelesion stages," American Journal of Pathology, vol. 95, no. 3, pp. 775-792, 1979.

[110] R. Natarajan, R. G. Gerrity, J. L. Gu, L. Lanting, L. Thomas, and J. L. Nadler, "Role of 12-lipoxygenase and oxidant stress in hyperglycaemia-induced acceleration of atherosclerosis in a diabetic pig model," Diabetologia, vol. 45, no. 1, pp. 125133, 2002.

[111] D. Hamamdzic, R. S. Fenning, D. Patel et al., "Akt pathway is hypoactivated by synergistic actions of diabetes mellitus and hypercholesterolemia resulting in advanced coronary artery disease," The American Journal of Physiology, vol. 299, no. 3, pp. H699-H706, 2010.

[112] Y. X. Wang, R. Fitch, and W. Li, "Reduction of cardiac functional reserve and elevation of aortic stiffness in hyperlipidemic Yucatan minipigs with systemic and coronary atherosclerosis," Vascular Pharmacology, vol. 39, no. 1-2, pp. 6976, 2002.

[113] J. R. Turk, K. K. Henderson, G. D. Vanvickle, J. Watkins, and M. H. Laughlin, "Arterial endothelial function in a porcine model of early stage atherosclerotic vascular disease," International Journal of Experimental Pathology, vol. 86, no. 5, pp. 335-345, 2005.

[114] B. J. de Smet, J. van der Zande, Y. J. Van der Helm, R. E. Kuntz, C. Borst, and M. J. Post, "The atherosclerotic Yucatan animal model to study the arterial response after balloon angioplasty: the natural history of remodeling," Cardiovascular Research, vol. 39, no. 1, pp. 224-232, 1998.

[115] M. F. Prescott, C. H. McBride, J. Hasler-Rapacz, J. Von Linden, and J. Rapacz, "Development of complex atherosclerotic lesions in pigs with inherited hyper-LDL cholesterolemia bearing mutant alleles for apolipoprotein B," The American Journal of Pathology, vol. 139, no. 1, pp. 139-147, 1991.

[116] J. Rapacz, J. Hasler-Rapacz, K. M. Taylor, W. J. Checovich, and A. D. Attie, "Lipoprotein mutations in pigs are associated with elevated plasma cholesterol and atherosclerosis," Science, vol. 234, no. 4783, pp. 1573-1577, 1986.

[117] J. Hasler-Rapacz, H. Ellegren, A. K. Fridolfsson et al., "Identification of a mutation in the low density lipoprotein receptor gene associated with recessive familial hypercholesterolemia in swine," American Journal of Medical Genetics, vol. 76, no. 5, pp. 379-386, 1998.

[118] J. Hasler-Rapacz, H. J. Kempen, H. M. Princen, B. J. Kudchodkar, A. Lacko, and J. Rapacz, "Effects of simvastatin on plasma lipids and apolipoproteins in familial hypercholesterolemic swine," Arteriosclerosis, Thrombosis, and Vascular Biology, vol. 16, no. 1, pp. 137-143, 1996.

[119] K. A. Grunwald, K. Schueler, P. J. Uelmen et al., "Identification of a novel Arg $\rightarrow$ Cys mutation in the LDL receptor that contributes to spontaneous hypercholesterolemia in pigs," Journal of Lipid Research, vol. 40, no. 3, pp. 475-485, 1999.

[120] C. Ahlstrom, K. Höglund, P. Hult, J. Häggström, C. Kvart, and P. Ask, "Assessing aortic stenosis using sample entropy of the phonocardiographic signal in dogs," IEEE Transactions on Biomedical Engineering, vol. 55, no. 8, pp. 2107-2109, 2008.

[121] J. G. Copeland, B. J. Maron, N. L. Luka, V. J. Ferrans, and L. L. Michaelis, "Experimental production of aortic valvular stenosis. Short-term and long-term studies in dogs," The Journal of Thoracic and Cardiovascular Surgery, vol. 67, no. 3, pp. 371-379, 1974.

[122] M. Shuvy, S. Abedat, R. Beeri et al., "Uraemic hyperparathyroidism causes a reversible inflammatory process of aortic valve calcification in rats," Cardiovascular Research, vol. 79, no. 3, pp. 492-499, 2008.

[123] V. Persy, A. Postnov, E. Neven et al., "High-resolution X-ray microtomography is a sensitive method to detect vascular calcification in living rats with chronic renal failure," Arteriosclerosis, Thrombosis, and Vascular Biology, vol. 26, no. 9, pp. 2110-2116, 2006.

[124] N. M. Rajamannan, E. Aikawa, and K. J. Grande-Allen, NHLBI Working Group Calcific Aortic Stenosis, National Heart Lung and Blood Institute, Chicago, Ill, USA, 2009.

[125] K. D. O’Brien, "Pathogenesis of calcific aortic valve disease: a disease process comes of age (and a good deal more)," Arteriosclerosis, Thrombosis, and Vascular Biology, vol. 26, no. 8, pp. 1721-1728, 2006.

[126] J. D. Miller, R. M. Weiss, and D. D. Heistad, "Calcific aortic valve stenosis: methods, models, and mechanisms," Circulation Research, vol. 108, no. 11, pp. 1392-1412, 2011.

[127] P. Sucosky, K. Balachandran, A. Elhammali, H. Jo, and A. P. Yoganathan, "Altered shear stress stimulates upregulation of endothelial VCAM-1 and ICAM-1 in a BMP-4- and TGFbeta1-dependent pathway," Arteriosclerosis, Thrombosis, and Vascular Biology, vol. 29, no. 2, pp. 254-260, 2009.

[128] K. Balachandran, P. Sucosky, H. Jo, and A. P. Yoganathan, "Elevated cyclic stretch alters matrix remodeling in aortic valve cusps: implications for degenerative aortic valve disease," American Journal of Physiology, vol. 296, no. 3, pp. H756-H764, 2009.

[129] C. Y. Yip and C. A. Simmons, "The aortic valve microenvironment and its role in calcific aortic valve disease," Cardiovascular Pathology, vol. 20, no. 3, pp. 177-182, 2011.

[130] J. T. Butcher, C. A. Simmons, and J. N. Warnock, "Mechanobiology of the aortic heart valve," The Journal of Heart Valve Disease, vol. 17, no. 1, pp. 62-73, 2008.

[131] K. Balachandran, S. Konduri, P. Sucosky, H. Jo, and A. P. Yoganathan, "An ex vivo study of the biological properties of porcine aortic valves in response to circumferential cyclic stretch," Annals of Biomedical Engineering, vol. 34, no. 11, pp. 1655-1665, 2006.

[132] W. D. Merryman, H. Y. Shadow Huang, F. J. Schoen, and M. S. Sacks, "The effects of cellular contraction on aortic valve leaflet flexural stiffness," Journal of Biomechanics, vol. 39, no. 1, pp. 88-96, 2006. 
[133] W. D. Merryman, H. D. Lukoff, R. A. Long, G. C. Engelmayr, R. A. Hopkins, and M. S. Sacks, "Synergistic effects of cyclic tension and transforming growth factor-betal on the aortic valve myofibroblast," Cardiovascular Pathology, vol. 16, no. 5, pp. 268-276, 2007.

[134] J. A. Stella, J. Liao, and M. S. Sacks, "Time-dependent biaxial mechanical behavior of the aortic heart valve leaflet," Journal of Biomechanics, vol. 40, no. 14, pp. 3169-3177, 2007.

[135] E. H. Stephens, N. de Jonge, M. P. McNeill, C. A. Durst, and K. J. Grande-Allen, "Age-related changes in material behavior of porcine mitral and aortic valves and correlation to matrix composition," Tissue Engineering. Part A, vol. 16, no. 3, pp. 867-878, 2010.

[136] I. El-Hamamsy, K. Balachandran, M. H. Yacoub, L. M. Stevens et al., "Endothelium-dependent regulation of the mechanical properties of aortic valve cusps," Journal of the American College of Cardiology, vol. 53, no. 16, pp. 14481455, 2009.

[137] K. L. Billiar and M. S. Sacks, "Biaxial mechanical properties of the natural and glutaraldehyde treated aortic valve cuspPart I: experimental results," Journal of Biomechanical Engineering, vol. 122, no. 1, pp. 23-30, 2000.

[138] I. Vesely and R. Noseworthy, "Micromechanics of the fibrosa and the ventricularis in aortic valve leaflets," Journal of Biomechanics, vol. 25, no. 1, pp. 101-113, 1992.

[139] J. A. Stella and M. S. Sacks, "On the biaxial mechanical properties of the layers of the aortic valve leaflet," Journal of Biomechanical Engineering, vol. 129, no. 5, pp. 757-766, 2007.

[140] R. Zhao, K. L. Sider, and C. A. Simmons, "Measurement of layer-specific mechanical properties in multilayered biomaterials by micropipette aspiration," Acta Biomaterialia, vol. 7, no. 3, pp. 1220-1227, 2011.

[141] K. Balachandran, P. Sucosky, H. Jo, and A. P. Yoganathan, "Elevated cyclic stretch induces aortic valve calcification in a bone morphogenic protein-dependent manner," The American Journal of Pathology, vol. 177, no. 1, pp. 49-57, 2010.

[142] M. O. Platt, Y. Xing, H. Jo, and A. P. Yoganathan, "Cyclic pressure and shear stress regulate matrix metalloproteinases and cathepsin activity in porcine aortic valves," The Journal of Heart Valve Disease, vol. 15, no. 5, pp. 622-629, 2006.

[143] P. Thayer, K. Balachandran, S. Rathan et al., "The effects of combined cyclic stretch and pressure on the aortic valve interstitial cell phenotype," Annals of Biomedical Engineering, vol. 39, no. 6, pp. 1654-1667, 2011.

[144] J.-H. Chen and C. A. Simmons, "Cell-matrix interactions in the pathobiology of calcific aortic valve disease: critical roles for matricellular, matricrine, and matrix mechanics cues," Circulation Research, vol. 108, no. 12, pp. 1510-1524, 2011.

[145] V. K. Krishnamurthy, F. Guilak, D. A. Narmoneva, and R. B. Hinton, "Regional structure-function relationships in mouse aortic valve tissue," Journal of Biomechanics, vol. 44, no. 1, pp. 77-83, 2011.

[146] J. R. Hove, R. W. Köster, A. S. Forouhar, G. Acevedo-Bolton, S. E. Fraser, and M. Gharib, "Intracardiac fluid forces are an essential epigenetic factor for embryonic cardiogenesis," Nature, vol. 421, no. 6919, pp. 172-177, 2003.

[147] R. B. Hinton and K. E. Yutzey, "Heart valve structure and function in development and disease," Annual Review of Physiology, vol. 73, pp. 29-46, 2011.

[148] M. Briand, I. Lemieux, J. G. Dumesnil et al., "Metabolic syndrome negatively influences disease progression and prognosis in aortic stenosis," Journal of American College of Cardiology, vol. 47, no. 11, pp. 2229-2236, 2006.
[149] W. S. Aronow, K. S. Schwartz, and M. Koenigsberg, "Correlation of serum lipids, calcium, and phosphorus, diabetes mellitus and history of systemic hypertension with presence or absence of calcified or thickened aortic cusps or root in elderly patients," The American Journal of Cardiology, vol. 59, no. 9, pp. 998-999, 1987.

[150] K. Akat, J. J. Kaden, F. Schmitz et al., "Calcium metabolism in adults with severe aortic valve stenosis and preserved renal function," American Journal of Cardiology, vol. 105, no. 6, pp. 862-864, 2010.

[151] F. Civeira, "Guidelines for the diagnosis and management of heterozygous familial hypercholesterolemia," Atherosclerosis, vol. 173, no. 1, pp. 55-68, 2004.

[152] U. Schwarz, M. Buzello, E. Ritz et al., "Morphology of coronary atherosclerotic lesions in patients with end-stage renal failure," Nephrology, Dialysis, Transplantation, vol. 15, no. 2, pp. 218-223, 2000.

[153] D. R. Pomaro, S. S. Ihara, L. E. Pinto et al., "High glucose levels abolish antiatherosclerotic benefits of ACE inhibition in alloxan-induced diabetes in rabbits," Journal of Cardiovascular Pharmacology, vol. 45, no. 4, pp. 295-300, 2005.

[154] L. A. Cuniberti, P. G. Stutzbach, E. Guevara, G. G. Yannarelli, R. P. Laguens, and R. R. Favaloro, "Development of mild aortic valve stenosis in a rabbit model of hypertension," Journal of American College of Cardiology, vol. 47, no. 11, pp. 2303-2309, 2006.

[155] H. Zheng, C. Zhang, W. Yang et al., "Fat and cholesterol diet induced lipid metabolic disorders and insulin resistance in rabbit," Experimental and Clinical Endocrinology and Diabetes, vol. 117, no. 8, pp. 400-405, 2009.

[156] Y. Mitsuguchi, T. Ito, and K. Ohwada, "Pathologic findings in rabbit models of hereditary hypertriglyceridemia and hereditary postprandial hypertriglyceridemia," Comparative Medicine, vol. 58, no. 5, pp. 465-480, 2008.

[157] D. Versari, M. Gossl, D. Mannheim et al., "Hypertension and hypercholesterolemia differentially affect the function and structure of pig carotid artery," Hypertension, vol. 50, no. 6, pp. 1063-1068, 2007.

[158] E. Chérin, R. Williams, A. Needles et al., "Ultrahigh frame rate retrospective ultrasound microimaging and blood flow visualization in mice in vivo," Ultrasound in Medicine and Biology, vol. 32, no. 5, pp. 683-691, 2006.

[159] F. S. Foster, M. Y. Zhang, Y. Q. Zhou et al., "A new ultrasound instrument for in vivo microimaging of mice," Ultrasound in Medicine and Biology, vol. 28, no. 9, pp. 1165-1172, 2002.

[160] Y. Q. Zhou, F. S. Foster, D. W. Qu, M. Zhang, K. A. Harasiewicz, and S. L. Adamson, "Applications for multifrequency ultrasound biomicroscopy in mice from implantation to adulthood," Physiological Genomics, vol. 10, no. 2, pp. 113-126, 2002.

[161] Y. Q. Zhou, F. S. Foster, B. J. Nieman, L. Davidson, X. J. Chen, and R. M. Henkelman, "Comprehensive transthoracic cardiac imaging in mice using ultrasound biomicroscopy with anatomical confirmation by magnetic resonance imaging," Physiological Genomics, vol. 18, pp. 232-244, 2004.

[162] C. M. Otto, "Calcific aortic stenosis-time to look more closely at the valve," The New England Journal of Medicine, vol. 359, no. 13, pp. 1395-1398, 2008.

[163] S. J. Cowell, D. E. Newby, R. J. Prescott et al., "A randomized trial of intensive lipid-lowering therapy in calcific aortic stenosis," The New England Journal of Medicine, vol. 352, no. 23, pp. 2389-2397, 2005. 
[164] L. M. Moura, S. F. Ramos, J. L. Zamorano et al., "Rosuvastatin affecting aortic valve endothelium to slow the progression of aortic stenosis," Journal of the American College of Cardiology, vol. 49, no. 5, pp. 554-561, 2007.

[165] A. B. Rossebø, T. R. Pedersen, K. Boman et al., "Intensive lipid lowering with simvastatin and ezetimibe in aortic stenosis," The New England Journal of Medicine, vol. 359, no. 13, pp. 1343-1356, 2008.

[166] K. L. Chan, K. Teo, J. G. Dumesnil, A. Ni, and J. Tam, "Effect of lipid lowering with rosuvastatin on progression of aortic stenosis: results of the aortic stenosis progression observation: measuring effects of rosuvastatin (astronomer) trial," Circulation, vol. 121, no. 2, pp. 306-314, 2010.

[167] E. R. Mohler III, "Mechanisms of aortic valve calcification," American Journal of Cardiology, vol. 94, no. 11, pp. 13961402, 2004.

[168] K. D. O’Brien, J. L. Probstfield, M. T. Caulfield et al., "Angiotensin-converting enzyme inhibitors and change in aortic valve calcium," Archives of Internal Medicine, vol. 165, no. 8, pp. 858-862, 2005.

[169] R. Rosenhek, F. Rader, N. Loho et al., "Statins but not angiotensin-converting enzyme inhibitors delay progression of aortic stenosis," Circulation, vol. 110, no. 10, pp. 1291-1295, 2004.

[170] R. A. H. Stewart, A. J. Kerr, B. R. Cowan et al., "A randomized trial of the aldosterone-receptor antagonist eplerenone in asymptomatic moderate-severe aortic stenosis," American Heart Journal, vol. 156, no. 2, pp. 348-355, 2008. 


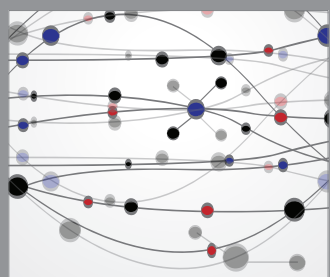

The Scientific World Journal
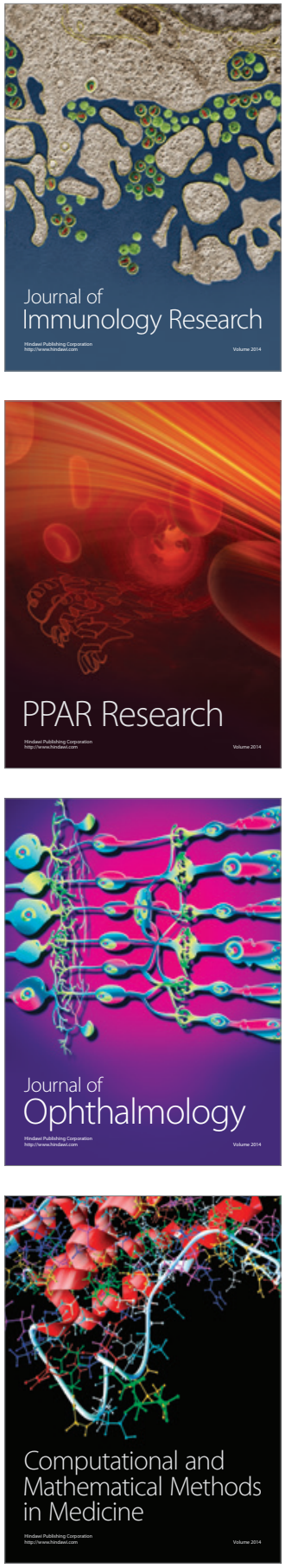

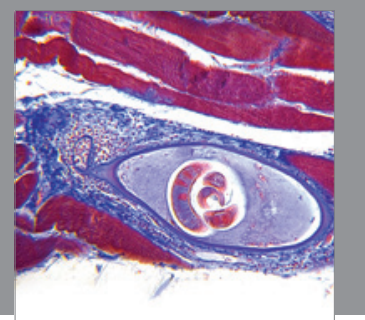

Gastroenterology

Research and Practice
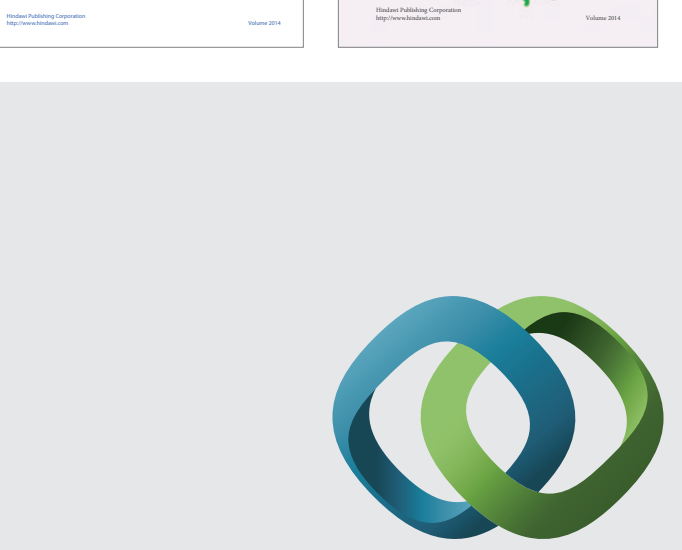

\section{Hindawi}

Submit your manuscripts at

http://www.hindawi.com
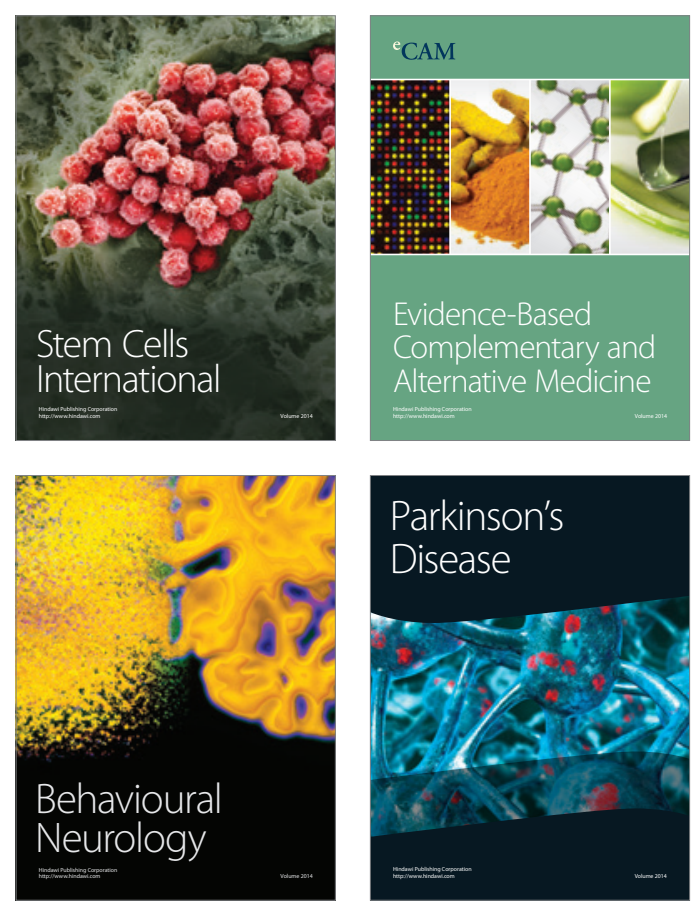

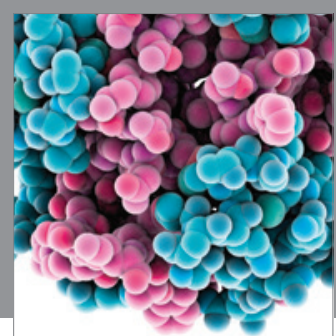

Journal of
Diabetes Research

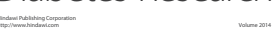

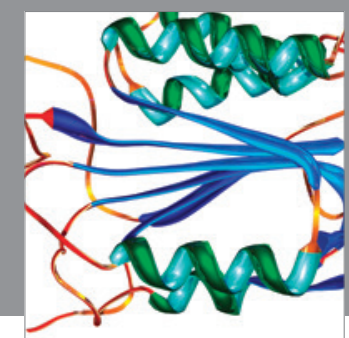

Disease Markers
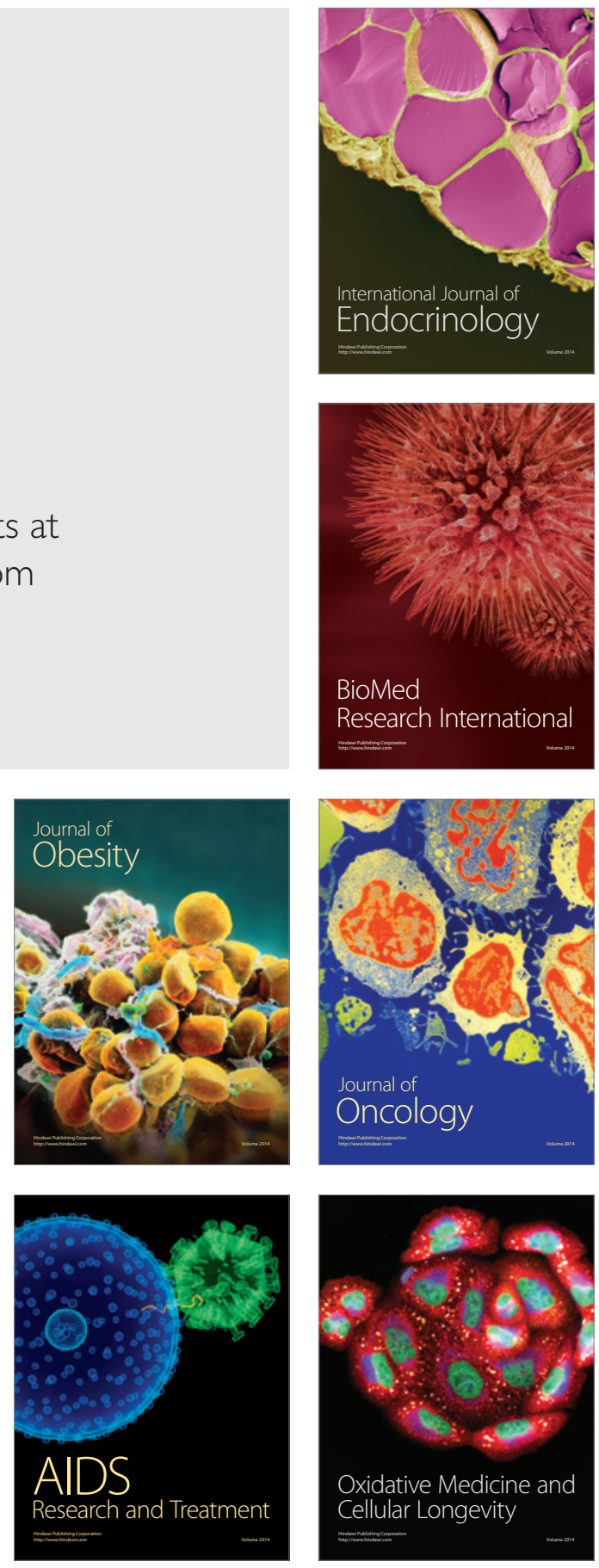